\title{
Clusters, asters and collective oscillations in chemotactic colloids
}

\author{
Suropriya Saha ${ }^{1}$, Ramin Golestanian ${ }^{2}$ and Sriram Ramaswamy ${ }^{1,3}$ \\ 1 Department of Physics, Indian Institute of Science, Bangalore 560 012, India \\ 2 Rudolf Peierls Center for Theoretical Physics, \\ University of Oxford, 1 Keble Road, \\ Oxford OX1 3NP, United Kingdom and \\ 3 TIFR Centre for Interdisciplinary Sciences, \\ 21 Brundavan Colony, Osman Sagar Road, \\ Narsingi, Hyderabad 500 075, India
}

\begin{abstract}
The creation of synthetic systems that emulate the defining properties of living matter, such as motility, gradient-sensing, signalling and replication, is a grand challenge of biomimetics. Such imitations of life crucially contain active components that transform chemical energy into directed motion. These artificial realizations of motility point in the direction of a new paradigm in engineering, through the design of emergent behavior by manipulating properties at the scale of the individual components. Catalytic colloidal swimmers are a particularly promising example of such systems. Here we present a comprehensive theoretical description of gradient-sensing of an individual swimmer, leading controllably to chemotactic or anti-chemotactic behavior, and use it to construct a framework for studying their collective behavior. We find that both the positional and the orientational degrees of freedom of the active colloids can exhibit condensation, signalling formation of clusters and asters. The kinetics of catalysis introduces a natural control parameter for the range of the interaction mediated by the diffusing chemical species. For various regimes in parameter space in the long-ranged limit our system displays precise analogs to gravitational collapse, plasma oscillations and electrostatic screening. We present prescriptions for how to tune the surface properties of the colloids during fabrication to achieve each type of behavior.
\end{abstract}

PACS numbers: 


\section{INTRODUCTION}

Dynamic self-organization of motile components can be observed in a wide range of length scales, from bird flocks [1] to bacterial colonies [2, 3] and assemblies of motor and structural proteins [4, 5]. The fascination with these phenomena has naturally inspired researchers to use a physical understanding of motility to engineer complex emergent behaviors in model systems that promise revolutionary advance in technological applications if combined with other novel biomimetic functions, such as signal processing and decision making [6], or replication [7].

Symmetry-based phenomenological theories, coarse-grained or particle-based [8 [14] offer a guide to the rich possibilities immanent in self-driven systems, but designing a system requires a bottom-up approach. Biological components pose inevitable limitations on this task, while chemical [15], mechanical [16] or externally actuated [17] imitations appear more promising. In addition to motility, living organisms have developed mechanisms that allow them to orient their motion in response to chemical gradients, and send signals to recruit or repel others [4, 18]. Can inanimate matter imitate these more complex functions? We show that it can, and present the necessary design principles. We consider the case of catalytic active colloids [19 22], which we now describe in brief. Recall that a colloidal particle can be driven "phoretically" into motion by externally imposed chemical, electrostatic or thermal gradients [23]. An active colloid - a particle coated asymmetrically with catalyst and immersed in a uniform background of substrate [53] - generates its own chemical gradient [19 22, 24, 25], and thus moves autonomously in a direction determined by the polarity of the coat [54]. Such self-phoretic particles, whose individual activity and interactions one can design, offer the opportunity to create systems with controllable, emergent collective behavior [29 37]. To this end it is essential to construct a description at a coarse-grained level, with coefficients expressed in terms of single-particle parameters 55 ].

Our focus is on how the center of mass and orientation vector of an active colloid are affected by an externally imposed gradient of substrate molecules. Depending on details of geometry, activity, and mobility [25], an active colloid will respond to the local gradient of the substrate concentration through four distinct mechanisms. (i) Chemotaxis: The fluid flows set up around the particle can turn its axis of orientation to align parallel or antiparallel to the local gradient; this process has active contributions arising from the chemical reaction as well as passive ones (ii) Polar run-and-tumble motion: The enzymatic rate depends nonlinearly on the local concentration of the substrate with a characteristic Michaelis-Menten form inherited from the underlying catalytic kinetics of the reactions [38]. The combination of enhanced activity at high concentrations and randomized orientation acts to effectively populate the colloids in "slow" regions [39]. (iii) Apolar run-and-tumble motion: An active colloid can also chemotax by a net motion of its center along a gradient in a noise-averaged sense. (iv) Phoretic response: The colloid moves along an external chemical gradient by diffusiophoresis. A summary of the different modes is depicted in Fig. 1.

Catalytic colloids consume a substrate and generate product molecules, and hence act as mobile sources and sinks of these chemicals in the solution making their concentration profile nonuniform. In a suspension of such active colloids, each individual responds - via the above four mechanisms - to the gradients produced by other colloids due to their activities. The various contributions are independent of each other and their balance will be modified as we move in the space of control parameters, leading to a variety of collective behaviors. In particular, we highlight the intriguing possibility that the positional and orientational 


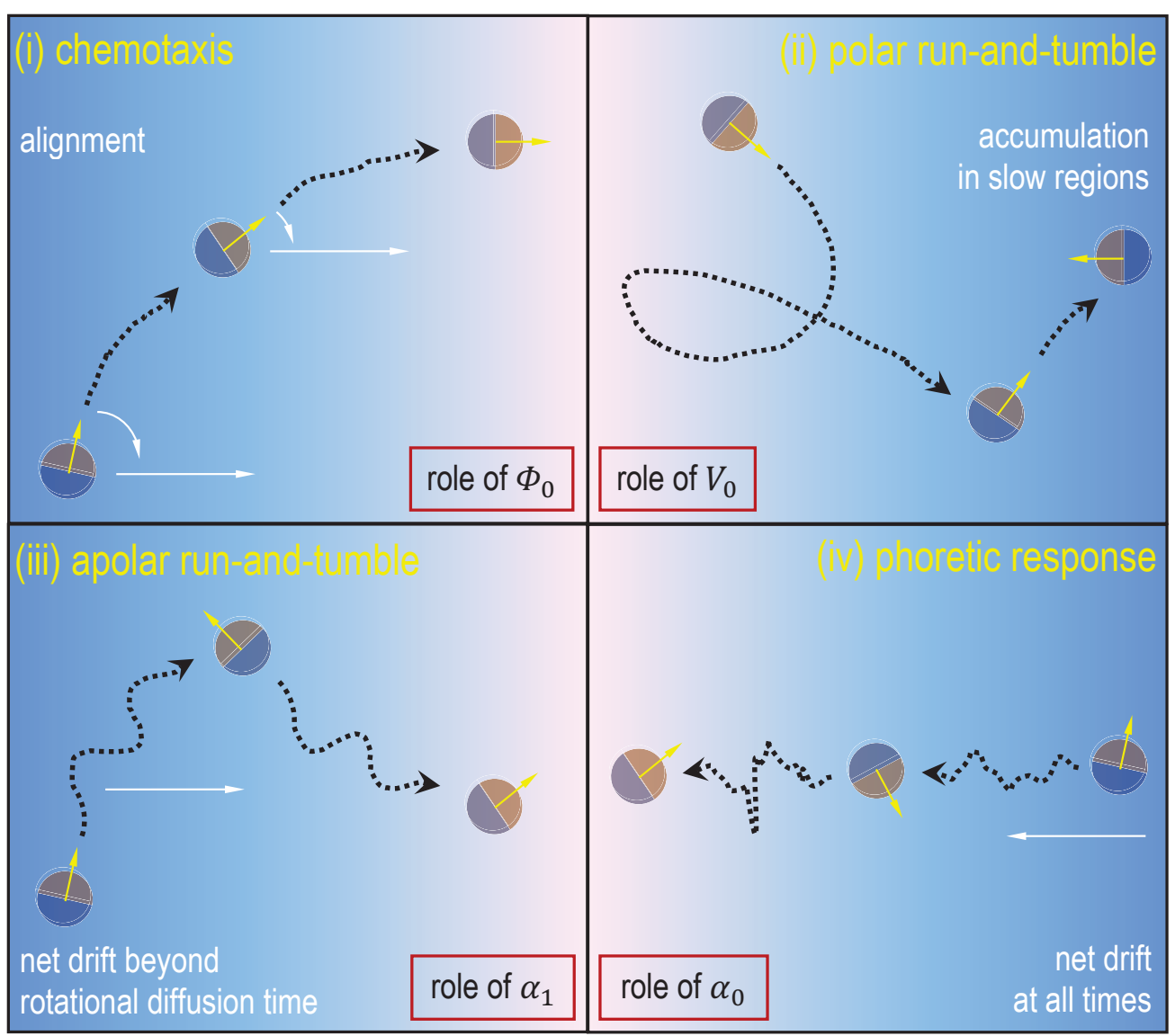

FIG. 1: A schematic summary of the four different ways a single swimmer responds to gradients corresponding to the different terms in Eq. 1.1. In each panel, three consecutive snapshots (with equal time intervals) are sketched together with typical connecting trajectories. In (i) and (ii) the polarity of the colloid controls the direction of motion. In (iii) the motion will be along the main symmetry axis of the colloid but driven by the gradient (hence the colloid can move forward or backward instantaneously). In (iv) the motion is independent of the polarity and symmetry axis of the colloid. Processes (i) and (iv) represent steady angular and linear drift while in (ii) and (iii) the gradient-seeking behavior is assisted by noise. Each mechanism is controlled by the relevant spherical harmonics coefficient of the material-dependent particle mobility and activity, which can be modified by construction.

degrees of freedom could exhibit different and independent types of order depending on the parameters, as shown in Figs. 2 and 3.

We consider a fluid medium containing a concentration $s(\mathbf{r}, t)$ of substrate $(\mathrm{S})$ molecules, which upon contact with a catalyst are converted to a product $\mathrm{P}$ with concentration $p(\mathbf{r}, t)$. The rate of conversion $\kappa$ obeys Michaelis-Menten kinetics [40], growing linearly at small $s$ and crossing over to saturation for sufficiently large values of $s$. Our main results are as follows: (1) for the case of a uniform gradient of substrate, we establish the form of the angular velocity $\boldsymbol{\omega}$ induced on a single catalytic colloid as a function of the spherical harmonic components of the activity $\sigma$ and the mobilities $\mu_{s}$ and $\mu_{p}$ corresponding to $\mathrm{S}$ and $\mathrm{P}$. We can therefore propose criteria for the surface patterning required to produce chemotactic 
and anti-chemotactic motion. We also find the various contributions to the translational velocity $\boldsymbol{v}$ of the colloid, arising from self-propulsion and drift due to the external gradient. Explicitly, we find

$$
\begin{aligned}
\boldsymbol{\omega} & =\Phi_{0}\left(\sigma, \mu_{p}, \mu_{s}\right) \hat{\mathbf{n}} \times \nabla s \\
\mathbf{v} & =V_{0}(s) \hat{\mathbf{n}}-\alpha_{0} \boldsymbol{\nabla} s-\alpha_{1} \hat{\mathbf{n}} \hat{\mathbf{n}} \cdot \nabla s .
\end{aligned}
$$

where the definitions of the coefficients in terms of the surface properties of the colloids are given below. (2) We use our results for a single particle in an external gradient to construct the collective equations of motion for the number density and orientation of the colloids in a uniform medium, interacting via their effect on the substrate and product concentration fields. The interplay between self-propulsion, phoretic drift, and alignment, driven by and mediated via chemicals, falls into two distinct regimes. (a) When the fuel concentration is small enough such that the catalytic activity is diffusion-limited, the chemical concentrations will be effectively screened, and the system could develop enhanced number fluctuations and clumping instabilities (where all wavelengths above a threshold are unstable) and patterns with a given length scale (where the fastest-growing mode has finite wavelength). (b) At sufficiently high fuel concentrations where the catalytic activity becomes reaction-limited, the chemical fields are not screened and can mediate long-ranged interactions that could lead to a wider variety of instabilities. In particular, for the case of effectively attractive phoretic interaction we observe collapse transitions that are dissipative analogs of a Jeans instability [43], with or without simultaneous condensation of asters. For effectively repulsive phoretic interactions, we observe counter-intuitively that a collapsed phase with aster condensation is still possible, as are stable phases exhibiting Debye-like screening, similar to electrolytes. Moreover, we find that in this regime the system could exhibit plasma-like oscillations in response to perturbations, or spontaneous, self-sustained ringing. Detailed phase diagrams inferred from our stability analysis and structure factor calculations are found in Figs. 2 and 3. These are parameterized, via definitions in Eq. 2.5 below, by coefficients $A$ describing the chemotactic response from panels (i) and (ii) of Fig. 1, and $B$ the phoretic response from panels (iii) and (iv) of that figure. We now show how we obtained these results.

\section{A. Background: diffusiophoresis and self-diffusiophoresis}

Diffusiophoresis [56] is the force-free, torque-free propulsion of a colloid by a solute concentration gradient [23]. In a fluid of viscosity $\eta$ at temperature $T$ a species with concentration $c$ interacting through an effective potential $\Psi$ with a particle surface with normal along the local $z$ axis gives rise, via the Stokes equation, to a surface "slip velocity" $\mathbf{v}_{\text {slip }}=\mu \nabla_{\|} c$, with

the phoretic mobility $\mu=\frac{k_{B} T}{\eta} \int_{0}^{\infty} z\left(1-e^{-\Psi / k_{B} T}\right) d z$ that can have either sign depending on $\Psi$ [41]. We are also interested here in self-diffusiophoresis [20] that occurs when $\nabla c$ is not imposed externally but generated by processes on the particle itself.

\section{B. A single chemotactic motile colloid}

Consider a single swimmer, whose mobilities and catalytic coat have the common symmetry axis $\hat{\mathbf{n}}$. When placed in a uniform substrate background, such a particle moves in a direction determined by $\hat{\mathbf{n}}$ if the coatings are sufficiently asymmetric [25]. What happens 


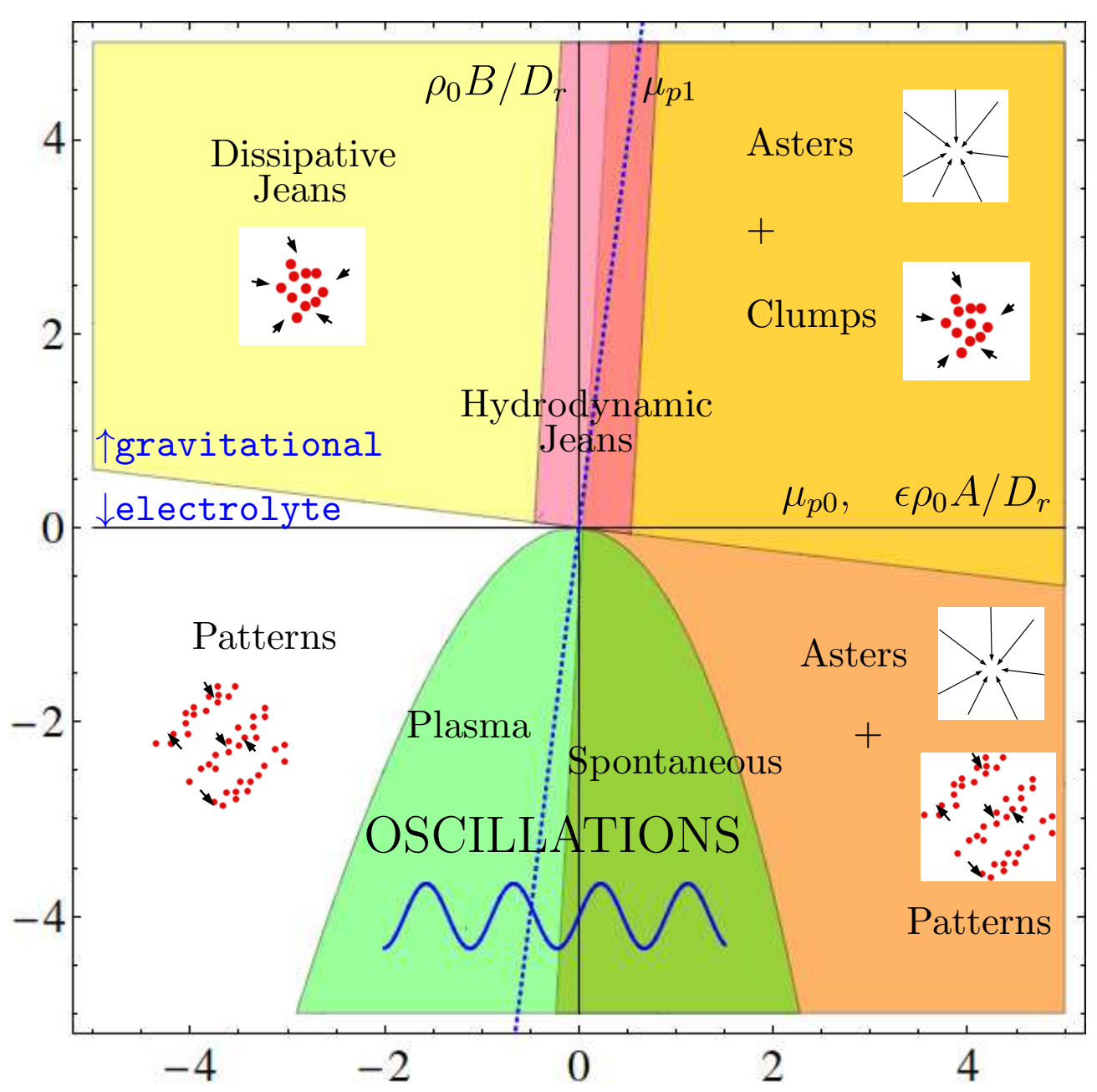

FIG. 2: The phase diagram in the reaction-limited regime (with abundant fuel) shows a variety of possible states in the parameter space spanned by suitably non-dimensionalized effective chemotactic $(A)$ and phoretic $(B)$ response coefficients, defined in Eq. 2.5. The dashed line and the $A$-axes correspond to independent changes of the fundamental and first harmonic $\left(\mu_{p 0}\right.$ and $\left.\mu_{p 1}\right)$ of the mobility corresponding to the reaction product, respectively. They represent possible experimental paths that can be explored in sequences of experiments on particles designed with suitable mobility coats.

in an inhomogeneous background? Can the flows set up by the interaction of $\mathrm{S}$ and $\mathrm{P}$ with the swimmer surface reorient its axis $\hat{\mathbf{n}}$ with respect to the local concentration gradient, thus imitating chemotaxis? To answer this question, we solve for the concentrations $s$ and $p$, with diffusivities $D_{s}$ and $D_{p}$ respectively. We incorporate the catalytic chemical reaction $\mathrm{S} \rightarrow \mathrm{P}$ through source and sink boundary conditions on particle fluxes normal $(\perp)$ to the swimmer surface:

$$
-D_{s} \nabla_{\perp} s=-\kappa_{1} s P_{s} \sigma(\theta, \phi) ; \quad-D_{p} \nabla_{\perp} p=\kappa_{2} P_{p} \sigma(\theta, \phi),
$$

where $P_{p}(\theta, \phi) \equiv 1-P_{s}(\theta, \phi)$ is the probability that the enzyme at $(\theta, \phi)$ is bound to the substrate. Stationarity implies $\kappa_{1} s P_{s}=\kappa_{2} P_{p}$ leading to the Michaelis-Menten [40] expression $\kappa_{2} P_{p} \equiv \kappa(s) \equiv \kappa_{2} \kappa_{1} s /\left(\kappa_{2}+\kappa_{1} s\right)$ for the reaction velocity per molecule. Number conservation 
for the products and substrates, and the assumption that $s$ and $p$ diffuse rapidly compared to the colloid so that time dependencies and advection by flow [42] can be ignored give $D_{p} p+D_{s} s=D_{s} s_{b}$, where $s_{b}$ is the background substrate profile. We thus need to solve for just one of the two concentration fields. We work in the linear regime [57] $s_{b} \ll \kappa_{1} / \kappa_{2}$, where the profile of product $p$ resulting from this process is sensitive to the imposed gradient of $s$, and in the limit where $\mathrm{S}$ diffuses rapidly so that its profile is maintained. The resulting slip velocity, which has contributions from both the substrate and the product, leads to the linear and angular velocities $\boldsymbol{\omega}=-\frac{3}{16 \pi R} \int \hat{\mathbf{r}} \times \mathbf{v}_{\text {slip }}(\mathbf{r}) \mathrm{d} \Omega$ and $\mathbf{v}=-\frac{1}{4 \pi} \int \mathbf{v}_{\text {slip }}(\mathbf{r}) \mathrm{d} \Omega$ for spherical colloids.

To understand the general trends in chemotactic behavior arising from simple catalytic patterns, we work with a limited number of non-zero spherical harmonic components of $\sigma$ and $\mu_{p}$. For example, taking $\sigma_{l}, \mu_{p l}=0$ for $l \geq 3$ we find the expression for the angular velocity given in Eq. 1.1 with

$$
\Phi_{0}=-\frac{3 \mu_{s 1}}{4 R}-\frac{\kappa_{1}}{60 D_{p}}\left(5 \mu_{p 1} \sigma_{0}+2 \mu_{p 2} \sigma_{1}-\mu_{p 1} \sigma_{2}\right)
$$

where a negative (positive) value corresponds to chemotactic (antichemotactic) response. The first term on the left is the passive response to the external gradient due to a polarity in $\mu_{s}$ alone while the second term is the active contribution involving both $\sigma$ and $\mu_{p}$. The form of Eq. 1.3 serves to illustrate some features that hold even without the truncated expansion in $l$ : if either $\sigma$ or $\mu_{p}$ contain all odd or all even harmonics there is no reorientation in response to the gradient, a result which holds for spheroidal swimmers as well. The expression for the product contribution in Eq. 1.3 is a sum of products of $\sigma_{l}$ and $\mu_{p, l \pm 1}$, which can be used to design chemotactic colloids with a desired response. Lastly, regardless of the form of $\sigma$, $\omega=0$ for $\mu_{p}$ uniform over the sphere.

We also obtain the net translational velocity $\mathbf{v}$, as in Eq. 1.1 where

$$
\begin{aligned}
V_{0} & =\frac{\kappa_{1} s_{b}}{15 D_{p}}\left(5 \sigma_{1} \mu_{p 0}+2 \sigma_{2} \mu_{p 1}-\sigma_{1} \mu_{p 2}\right), \\
\alpha_{0} & =-\left(\mu_{s 0}+\frac{1}{10} \mu_{s 2}\right)-\frac{\kappa_{1} R}{10 D_{p}}\left(\sigma_{0} \mu_{p 2}-\frac{2}{9} \sigma_{1} \mu_{p 1}-2 \sigma_{2} \mu_{p 0}\right. \\
& \left.+\frac{1}{35} \sigma_{2} \mu_{p 2}\right), \\
\alpha_{1} & =-\frac{1}{10} \mu_{s 2}-\frac{\kappa_{1} R}{30 D_{p}}\left(10 \sigma_{0} \mu_{p 0}+\sigma_{0} \mu_{p 2}+2 \sigma_{1} \mu_{p 1}-2 \sigma_{2} \mu_{p 0}\right. \\
& \left.+\frac{29}{35} \sigma_{2} \mu_{p 2}\right) .
\end{aligned}
$$

The three contributions to the translational velocity correspond to self-propulsion (along $\hat{\mathbf{n}}$ ), phoretic drift (along $\nabla s$ ), and an anisotropic drift that is instantaneously along $\hat{\mathbf{n}}$, but leads to net motion along $\nabla s$ as rotational noise de-correlates $\hat{\mathbf{n}}$. The latter amounts to a contribution to run-and-tumble gradient-seeking motion, which we name apolar run-andtumble; see Fig. 1.

To demonstrate how the chemotactic response of catalytic colloids can be designed, we have calculated $\Phi_{0}$ for an example of swimmers with uniform spheroidal caps of catalytic and mobility patterns as $\sigma(\theta) \propto \Theta\left(\theta-\theta_{1}\right)$ and $\mu_{p} \propto 1+\Theta\left(\theta-\theta_{2}\right)$. Figure 4 shows $\Phi_{0}$ as a function of $\theta_{1}$ for different values of $\theta_{2}$. For $\theta_{2}=\pi / 2, \Phi_{0}$ is antisymmetric as a function 


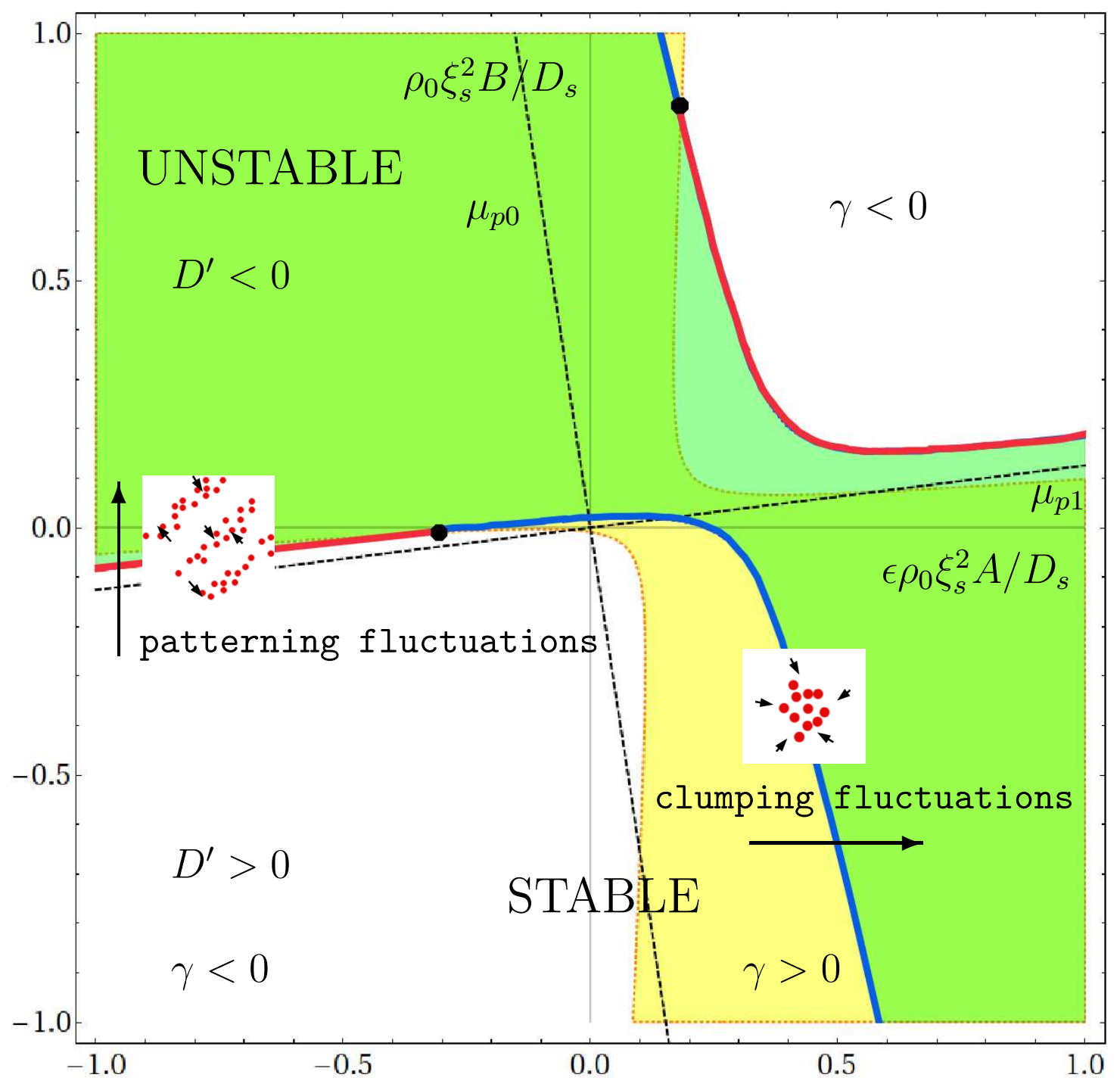

FIG. 3: The diffusion-limited regime (with limited fuel supply) exhibits different types of instability in the parameter space spanned by suitably non-dimensionalized effective chemotactic $(A)$ and phoretic $(B)$ response coefficients, defined in Eq. 2.5. The effective diffusivity $D^{\prime}$ (defined in Eq. 3.2) is negative in the green region and positive in the white or yellow regions, signalling the presence of an instability upon going from the yellow or white to green. The dominant fluctuations on approaching the instability signal a tendency to form modulations (across the red line) or clumps (across the blue line) state, depending on the sign of the parameter $\gamma$ (defined in Eq. 3.3). The character of the fluctuations on the stability boundary changes at the location shown by the black dot. The axes corresponding to possible experimental changes of the fundamental and first harmonic $\left(\mu_{p 0}\right.$ and $\left.\mu_{p 1}\right)$ of the mobility corresponding to the reaction product, respectively, are shown by two dashed lines. 
of $\theta_{1}$. For a given $\theta_{2}, \Phi_{0}$ peaks near $\theta_{1}=\theta_{2}$ as the slip velocity is maximum when the position where $\mu_{p}$ is maximum coincides with the region where $p$ changes most rapidly, which for the given form of $\sigma$ is at $\theta_{1}$. This example showcases the possibility to control the response of individual catalytic colloids, and thus their collective behaviors, by following design rules that include varying systematically their geometric features. We now combine the individual responses of active colloids to construct a theoretical description for their collective behaviors.

\section{FROM CHEMOTAXIS TO COLLECTIVE MOTION}

Catalytic swimmers of the type discussed above interact through the $\mathrm{S}$ and $\mathrm{P}$ chemical fields as well as via hydrodynamics [44-46]. We restrict our attention to their chemotactic interaction, and construct the collective behavior of many swimmers by looking at pairwise interactions. Consider, therefore, two swimmers separated by a distance $r$ in a uniform medium of substrate molecules. The reaction $\mathrm{S} \rightarrow \mathrm{P}$ that takes place on the surface of each swimmer modifies the $s$ field as seen by the others, and each is also a source for $\mathrm{P}$. In the absence of a background of other swimmers, inhomogeneities in the $s$ and $p$ fields in steady state decay as $1 / r$. Each swimmer senses and responds to the magnitude and the gradient of $s$ through the motility and chemotaxis mechanisms outlined above. In addition, each particle responds to the $p$ field produced by the reactions on the surfaces of all the particles, just as it would to any externally imposed solute gradient [23]. The resulting equations of motion for the position $\mathbf{r}_{\alpha}$ and orientation unit vector $\hat{\mathbf{n}}_{\alpha}$ of the $\alpha$ th, to linear order in $\nabla s$, $\nabla p$, take the form

$$
\begin{aligned}
\frac{\mathrm{d} \mathbf{r}_{\alpha}}{\mathrm{d} t}= & V_{0}(s) \hat{\mathbf{n}}_{\alpha}-\alpha_{0} \nabla s-\alpha_{1} \hat{\mathbf{n}}_{\alpha} \hat{\mathbf{n}}_{\alpha} \cdot \nabla s+\beta_{0} \nabla p \\
& +\beta_{1} \hat{\mathbf{n}}_{\alpha} \hat{\mathbf{n}}_{\alpha} \cdot \nabla p+\sqrt{2 D} \mathbf{f}_{\alpha}^{r}(t), \\
\frac{\mathrm{d} \mathbf{n}_{\alpha}}{\mathrm{d} t}= & \Phi_{0}\left(\hat{\mathbf{n}}_{\alpha} \times \nabla s\right) \times \hat{\mathbf{n}}_{\alpha}+\Omega_{0}\left(\hat{\mathbf{n}}_{\alpha} \times \nabla p\right) \times \hat{\mathbf{n}}_{\alpha} \\
& +\sqrt{2 D_{r}} \hat{\mathbf{n}}_{\alpha} \times \mathbf{f}_{\alpha}^{n}(t),
\end{aligned}
$$

where additional coupling constants

$$
\Omega_{0}=-\frac{3 \mu_{p 1}}{4 R}, \quad \beta_{0}=-\left(\mu_{p 0}+\frac{1}{10} \mu_{p 2}\right), \quad \beta_{1}=-\frac{1}{10} \mu_{p 2},
$$

are introduced to take account of the response of each colloid to a product gradient produced by the others. In Eq. 2.1 thermal as well as active fluctuations are included phenomenologically via Gaussian unit-strength white noise terms $\mathbf{f}_{\alpha}^{r}, \mathbf{f}_{\alpha}^{n}$, with strengths $D$ and $D_{r}$. $\Phi_{0}>0$ and $\Omega_{0}>0$ correspond to swimmers that respond chemotactically to $\nabla s$ and $\nabla p$ respectively. $\alpha_{0}>0$ and $\beta_{0}>0$ imply attractive contributions to the interactions between the swimmers due to $s$ and $p$ respectively. $V_{0}(s)>0$ by definition as we choose $\hat{\mathbf{n}}_{\alpha}$ to point in the direction in which a solitary swimmer moves. The form of Eq. 2.1 follows on general grounds of symmetry. The point of our calculation is that it gives explicit expressions for the tactic and phoretic mobilities and the expressions for $\alpha_{i}$ and $\beta_{i}$. Moreover, the substrate and product fields are themselves determined by the distribution of colloid positions and orientations. The substrate is consumed and the product is generated at the rate $Q(\mathbf{r}, t)=\kappa(s) \sum_{\alpha} \int_{\left|\mathbf{X}_{\alpha}\right|=R} \delta\left(\mathbf{r}-\mathbf{r}_{\alpha}-\mathbf{X}_{\alpha}\right) \sigma\left(\mathbf{X}_{\alpha} \cdot \hat{\mathbf{n}}_{\alpha}\right)$, where $\mathbf{X}_{\alpha}$ is the position coordinate 


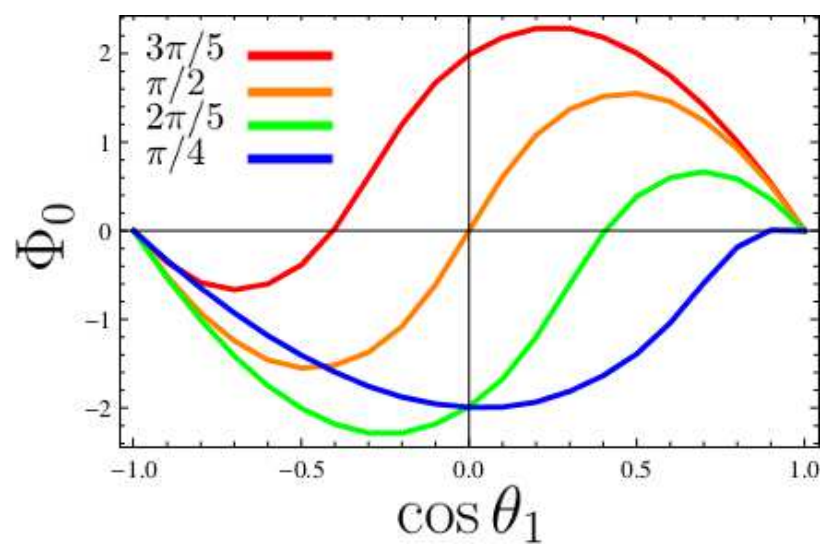

FIG. 4: Angular velocity coefficient for spherical colloids as a function of $\theta_{1}$ (that parameterizes the size of the catalytic coating) for different values of $\theta_{2}$ (that parameterizes the mobility pattern) quoted in the legend.

on the $\alpha$ th swimming sphere of radius $R$, and the catalytic coat $\sigma$ is expressed in lab-frame coordinates. We assume the system is maintained[58] in a steady state with mean substrate concentration $s_{0}$ and develop $Q$ to leading orders in a gradient expansion to obtain a coarsegrained description. We begin by relating the $s$ and $p$ fields to the coarse-grained density and orientation fields of the colloids, namely, $\sum_{\alpha} \delta\left(\mathbf{r}-\mathbf{r}_{\alpha}\right)=\rho(\mathbf{r})$ and $\sum_{\alpha} \hat{\mathbf{n}}_{\alpha} \delta\left(\mathbf{r}-\mathbf{r}_{\alpha}\right)=\mathbf{w}(\mathbf{r})$ (see the Supplementary Material). We find

$$
\left(\partial_{t}-D_{s} \nabla^{2}\right) s=-N \kappa(s)(\rho-\epsilon \nabla \cdot \mathbf{w})=-\left(\partial_{t}-D_{p} \nabla^{2}\right) p .
$$

In Eq. 2.3, $N=4 \pi R^{2} \sigma_{0}$ is the total number of enzymatic sites on the surface of the swimmer and $\epsilon=R \sigma_{1} / 3 \sigma_{0}$ measures the degree of polarity of the catalytic coat. We work in the limit of $\partial_{t} s=\partial_{t} p=0$. Linearizing Eq. 2.3 around a steady state with $\left(\rho_{0}, s_{0}, p_{0}\right)$, we find the following results for the Fourier components of the concentration at wavevector $\mathbf{q}: s_{\mathbf{q}}=-N \kappa\left(s_{0}\right)\left(\rho_{\mathbf{q}}-\epsilon i \mathbf{q} \cdot \mathbf{w}_{\mathbf{q}}\right) /\left[D_{s}\left(q^{2}+\xi_{s}^{-2}\right)\right], p_{\mathbf{q}}=-D_{s} s_{\mathbf{q}} / D_{p}$, where we introduce the screening length

$$
\xi_{s}=\left[N \rho_{0} \kappa^{\prime}\left(s_{0}\right) / D_{s}\right]^{-1 / 2},
$$

that is a measure of the range of interactions mediated by $\mathrm{S}$ and P. For $s_{0} \ll \kappa_{2} / \kappa_{1}$, i.e. on the linear or unsaturated part of the MM curve, $\xi_{s}$ is finite and the interactions are therefore short-ranged. For $s_{0} \gg \kappa_{2} / \kappa_{1}$, i.e. on the saturated part of the MM curve, $\xi_{s} \rightarrow \infty$ and the product mediates an effective long-ranged interaction amongst the colloids.

Starting from the Langevin equations in Eq. 2.1, we next construct equations of motion for $\rho$ and $\mathbf{n}(\mathbf{r})=\mathbf{w} / \rho$ as defined above Eq. 2.3. While coarse-graining, we see inevitably that the dynamical equation for $\mathbf{n}$ involves higher moments of the orientational distribution function, which must be re-expressed in terms of lower moments [47, 48] using an appropriate closure which we discuss in the Supplementary Material. The S and P fields produced by inhomogeneities in density and the divergence of the polar order parameter mediate interactions between swimmers through $\rho$ and the longitudinal component $\mathbf{n}_{L q}=\hat{\mathbf{q}} \hat{\mathbf{q}} \cdot \mathbf{n}_{q}$. The linearized dynamics in the isotropic phase closes in terms $\rho$ and $\mathbf{n}_{L \mathbf{q}}$, whose coarsegrained equations we present in the saturated limit and for wavenumbers $q \ll \xi_{s}^{-1}$ in the unsaturated case. We will see that despite the presence of a self-propelled velocity field 
and a density, there is an important contrast relative to models such as Toner-Tu [8]: the interactions considered here offer no mechanism to promote flocking, i.e., the global parallel alignment of $\mathbf{n}$.

We have calculated the mode structure and steady state structure factors $S_{\rho}=\int_{\omega}\left\langle\left|\rho_{\mathbf{q} \omega}\right|^{2}\right\rangle$ and $S_{n}=\int_{\omega}\left\langle\left|\mathbf{n}_{L \mathbf{q} \omega}\right|^{2}\right\rangle$ for both the unsaturated and the saturated cases by adding phenomenological gaussian white noise terms, conserving for $\rho$ and nonconserving for $\mathbf{n}_{L}$, to the equations of motion. The equations of motion and their stability analysis which we now present are best shown in terms of coefficients

$$
\begin{aligned}
& A=N \kappa\left(s_{0}\right)\left[\frac{\Omega_{0}}{D_{p}}-\frac{\Phi_{0}}{D_{s}}+\left.\frac{V_{0}\left(s_{0}\right)}{2 D_{s}} \frac{d \ln \kappa}{d s}\right|_{s_{0}}\right], \\
& B=N \kappa\left(s_{0}\right)\left[\frac{1}{D_{p}}\left(\beta_{0}+\frac{\beta_{1}}{3}\right)+\frac{1}{D_{s}}\left(\alpha_{0}+\frac{\alpha_{1}}{3}\right)\right],
\end{aligned}
$$

which give the effective chemotactic and phoretic response to gradients respectively.

\section{UNSATURATED}

In the unsaturated case $\xi_{s}<\infty$, for wavenumbers $q \ll \xi_{s}^{-1}$, coarse grained equations read

$$
\begin{aligned}
& {\left[\partial_{t}\right.}\left.+2 D_{r}-\left(D+\frac{v_{1}^{2} s_{0}^{2}}{30 D_{r}}\right) \nabla^{2}+\left(\epsilon \rho_{0} \xi_{s}^{2} A-\frac{v_{1}^{2} s_{0}^{2}}{90 D_{r}}\right) \nabla \nabla \cdot\right] \mathbf{n}_{L} \\
&+\frac{\left(v_{1} s_{0}-\rho_{0} \xi_{s}^{2} A\right)}{3 \rho_{0}} \nabla \rho=0 \\
& {\left[\partial_{t}-\left(D-\rho_{0} \xi_{s}^{2} B\right) \nabla^{2}\right] \rho_{0}^{-1} \delta \rho+\left(v_{1} s_{0}-\epsilon \rho_{0} \xi_{s}^{2} B \nabla^{2}\right) \nabla \cdot \mathbf{n}_{L}=0, }
\end{aligned}
$$

where we use $V_{0}\left(s_{0}\right) \equiv v_{1} s_{0}$ as the self-phoretic velocity scales linearly with the substrate concentration.

Several features of Eq. 3.1 are noteworthy. From Eq. 2.3, P is abundantly available or S is depleted where the density $\rho$ is high. Phoretic movement up (down) the gradient of $p(s)$ can thus lead to a propensity of swimmers to swim up their concentration gradients and hence a change in the sign of the diffusivity in Eq. 3.1b through $\rho_{0} \xi_{s}^{2} B$. Since the swimmer preferentially moves along its polar axis, $\mathbf{n}$ can be viewed as a velocity field and the $\nabla \rho$ term as a pressure gradient. We see that for large enough $\epsilon \rho_{0} \xi_{s}^{2} A$, the signs of coefficients that are analogous to bulk viscosity, and squared sound speed can change signalling an instability and possible novel condensation phenomena whose nature will be revealed only by a nonlinear treatment with appropriate noise terms. Note that $A$ contains two contributions: (i) chemotactic alignment with the local gradient in $s$ and $p$ and (ii) slowing down of swimmers due to increased substrate consumption as a result of a local excess of $\rho$ that depletes $s$ locally. This provides a realization of the density-dependent self-propulsion velocity of [49]. In the overdamped limit, for large $A$ and $B$, one eigenmode with relaxation

$$
-i \omega=-D^{\prime} q^{2} \equiv-\left(D+\frac{v_{1}^{2} s_{0}^{2}}{6 D_{r}}-\rho_{0} \xi_{s}^{2} B-\frac{v_{1} s_{0} \rho_{0} \xi_{s}^{2} A}{6 D_{r}}\right) q^{2},
$$

goes unstable with growth rate $\sim q^{2}$ at small $q$. Competition with stabilizing effects at larger $q$ will lead to a modulated growth morphology with a length scale determined by the 
wavenumber of peak growth $\sim\left|D^{\prime}\right|^{1 / 2}$. The other mode, controlled by $D_{r}$, remains stable for $q \rightarrow 0$, i.e., interactions do not promote flocking. Working at large $D_{r}$ also justifies the overdamped limit.

In the parameter range where these modes are stable, the steady-state static small- $q$ structure factor takes the form $S_{\rho} \propto 1 /\left(D^{\prime}+\gamma q^{2}\right)$ where

$$
\begin{aligned}
\gamma & =2 \xi_{s}^{2} D_{r}\left[\rho_{0} \xi_{s}^{2} B+\frac{\rho_{0} \xi_{s}^{2} A v_{1} s_{0}}{6 D_{r}}+\frac{1}{3} \epsilon \rho_{0} \xi_{s}^{2} B\left(v_{1} s_{0}-\rho_{0} \xi_{s}^{2} A\right)\right. \\
& +\left(D-\epsilon \rho_{0} \xi_{s}^{2} A\right)\left(D+\frac{2 v_{1}^{2} s_{0}^{2}}{45 D_{r}}-\rho_{0} \xi_{s}^{2} B\right) \\
& \left.+D^{\prime}\left(\rho_{0} \xi_{s}^{2} B+\epsilon \rho_{0} \xi_{s}^{2} A-2 D\right)\right] .
\end{aligned}
$$

For $\gamma>0$, as $D^{\prime} \rightarrow 0^{+}, S_{\rho}$ displays fluctuations with a correlation length $\sqrt{\gamma / D^{\prime}}$ that diverges as $D^{\prime} \rightarrow 0$, presaging the onset of clumping (see Fig. 3). Still in the linearly stable regime but with $\gamma<0$, an analysis to order $q^{4}$ shows that the system has a tendency towards patterning with a characteristic length scale $\sim|\gamma|^{-1 / 2}$, whose origin involves a competition between the chemotactic $(A)$ and phoretic $(B)$ response to gradients.

\section{SATURATED}

Next we consider the saturated limit $\xi_{s} \rightarrow \infty$, realized by working at saturation concentrations on the MM curve.It is useful to define $\mathbf{E}(\mathbf{r})=-\nabla \int_{\mathbf{r}^{\prime}} \rho\left(\mathbf{r}^{\prime}\right) /\left|\mathbf{r}-\mathbf{r}^{\prime}\right|$, which plays the role of an electric field in Eq. 4.1a below. Equation 2.3 then implies $\nabla s=N \kappa_{2}\left(\mathbf{E}-\epsilon \rho_{0} \mathbf{n}_{L}\right) / D_{s}$. In this limit the orientation and density fields satisfy [59]

$$
\begin{aligned}
& {\left[\partial_{t}-\left(D+\frac{v_{0}^{2}}{30 D_{r}}\right) \nabla^{2}\right] \mathbf{n}_{L}+\frac{v_{0}}{3 \rho_{0}} \nabla \rho+\frac{A}{3} \mathbf{E}} \\
& -\left[\frac{v_{0}^{2}}{90 D_{r}}-\frac{2 \epsilon N \kappa_{2} v_{0} \rho_{0}}{135 D_{r}}\left(\frac{\beta_{1}}{D_{p}}+\frac{\alpha_{1}}{D_{s}}\right)\right] \nabla \nabla \cdot \mathbf{n}_{L} \\
& +\left[\frac{2 \epsilon^{2} A^{2} \rho_{0}^{2}}{15 D_{r}} n_{L}^{2}-\frac{\epsilon A}{3} \rho_{0}+2 D_{r}\right] \mathbf{n}_{L}=0 \\
& \left(\partial_{t}-D \nabla^{2}\right) \rho+\rho_{0}\left(v_{0}+\epsilon \rho_{0} B\right) \nabla \cdot \mathbf{n}_{L}-\rho_{0} B \nabla \cdot \mathbf{E}=0
\end{aligned}
$$

where $v_{0} \equiv \lim _{s_{0} \gg \kappa_{2} / \kappa_{1}} V_{0}\left(s_{0}\right)$. The electric-field character of $\mathbf{E}$ is evident in Eq. 4.1a through the alignment term $\propto A$ and the Ohmic current $\propto B$ in Eq. 4.1b. Note that a large and positive $\epsilon \rho_{0} A / 3$ can destabilize the $n_{L}=0$ state leading - once higher order terms are taken into account - to a state of nonzero $\mathbf{n}_{L}$, i.e. a condensation of asters. The phenomenon is related to that reported in [50], with the important difference in our case of long-range interactions mediated by $\mathbf{E}$, as in [51], with a resemblance to gravitational collapse [43].

In the overdamped limit, i.e. for sufficiently large $D_{r}$, the relaxation rates of the eigenmodes are

$$
-i \omega=\left\{\begin{array}{c}
\frac{G}{2 D_{r}^{\prime}}-\left[2 D+\frac{v_{0}\left(v_{0}+\epsilon \rho_{0} B\right)}{3 D_{r}^{\prime}}\right] q^{2}, \\
-2 D_{r}^{\prime}+O\left(q^{2}\right),
\end{array}\right.
$$

where $D_{r}^{\prime}=D_{r}-\epsilon \rho_{0} A / 6$ represents a modified rotational diffusion, and $G=2 \rho_{0} B D_{r}+$ $\frac{1}{3} \rho_{0} A v_{0}$ is an effective control parameter for the nature of interaction between the swimmers. 
Equation 4.1a shows that in the saturated limit the effective long-ranged interaction between colloids (as mediated by $\mathrm{S}$ and $\mathrm{P}$ ) leads to non-vanishing relaxation rates at $q=0$ for both modes, notwithstanding the conservation law governing $\rho$.

For $G<0$, the swimmers interact with long-ranged repulsive interactions. The structure factor $S_{\rho}(q \rightarrow 0)=0$, as it is a ratio of the strength of fluctuations and the wavenumber independent relaxation rate, which is reminiscent of suppression of charge density fluctuations in electrolytes. Including terms of higher order in $q$ yields a density structure factor with a peak at $q \sim G^{1 / 4}$, which is characteristic of micro-phase separation (white region in Fig. 2). We see from Eq. 4.1a that for $G>0$ and large $D_{r}^{\prime}$ the isotropic state with uniform density is linearly unstable for small wavenumber $q$, including $q=0$. This effect is a dissipative analog of the gravitational Jeans instability [43], and is a consequence of the long-ranged attractive interaction (yellow). Related behavior has been predicted for thermophoretic colloids [51]. Letting $D_{r}^{\prime} \rightarrow 0$ by increasing $A$ and keeping $G>0$ brings the system out of the overdamped region where the relaxation of $\mathbf{n}$ slows down and it behaves like a velocity field. This behavior where the system resembles a gravitational system conserving momentum and displays an instability formally equivalent to the standard hydrodynamic Jeans instability [43] (magenta). Modes with wavenumbers larger than a crossover scale given by a competition between the interaction strength $G$ and a squared sound speed equivalent $v_{0}\left(v_{0}+\epsilon \rho_{0} B\right)$ for our system are oscillatory, whereas modes with smaller wavenumbers are too "massive" and collapse. On further tuning the parameters to approach $D_{r}^{\prime}<0$, notwithstanding the value of $G$, one anticipates an instability towards a spontaneously oscillating state (green). Restricting our attention to the stable case, we find a structure factor for $\mathbf{n}_{L}$ with a correlation length $\sim\left(D / D_{r}^{\prime}\right)^{1 / 2}$ that grows as $D_{r}^{\prime}$ decreases, indicating strong fluctuations towards aster formation. For small $D_{r}^{\prime}$ and $G<0$ the response shows "plasma oscillations" [52]

with frequency $\sim \sqrt{|G|}$. For $D_{r}^{\prime}<0$ (dark green), the system can also develop spontaneous oscillations, or ringing.

\section{SUMMARY}

A colloid patterned with catalyst and immersed in a maintained reactant medium is a minimal nonequilibrium particle, displaying directed motion and related behaviours ruled out at thermal equilibrium. We have determined theoretically the nature of patterning that will cause such an active colloid to reorient along and move up or down a gradient of chemical reactant, thus delineating the principles for the design of chemotactic self-phoretic particles. Coarse-graining the resulting Langevin equations for the position and polar axis of one particle, we discover the dynamics of the density and polar order parameter of a collection. The interplay of chemotaxis and phoresis leads to clumping and patterning at low reactant concentration; at high concentration, the slow decay of diffusing reactants and products yields analogues of electrostatic and gravitational phenomena - Debye screening, microphase separation, plasma oscillations and gravitational collapse. The interactions promote aster formation, not a flocking transition, and the instabilities mediated by the long-range diffusion fields have a character distinct from those the generic instability driven by the velocity field in Stokesian active liquid crystals. We look forward to experimental tests and, eventually, practical application of our predictions. 


\section{Acknowledgments}

RG and SR acknowledge HFSP grant RGP0061/2013 and a J C Bose Fellowship respectively, and both thank the Isaac Newton Institute for Mathematical Sciences where a part of this work was completed. SS thanks TCIS for hospitality.

[1] Cavagna A, Giardina I (2014) Bird Flocks as Condensed Matter. Annu Rev Cond Matt Phys 5.

[2] Ben-Jacob E, Cohen I, Levine H (2000) Cooperative self-organization of microorganisms. Adv Phys 49:395.

[3] Zhang HP, Be'er A, Florin EL, Swinney HL (2010) Collective motion and density fluctuations in bacterial colonies. PNAS 107:13626?13630.

[4] Huber F et al. (2013) Emergent complexity of the cytoskeleton: from single filaments to tissue. Adv Phys 62:1.

[5] Schweitzer F (2007) Brownian Agents and Active Particles: Collective Dynamics in the Natural and Social Sciences (Springer Series in Synergetics), Springer

[6] The SWARMS project webpage. www.swarms.org.

[7] Wang T et al. (2011) Self-replication of information-bearing nanoscale patterns. Nature 478: $225-228$.

[8] Toner J, Tu Y (1995) Long-Range Order in a Two-Dimensional Dynamical XY Model: How Birds Fly Together. Phys Rev Lett 75:4326-4329.

[9] Ramaswamy S (2010) The Mechanics and Statistics of Active Matter. Annual Reviews 1: 323-345.

[10] Marchetti MC et al. (2013) Hydrodynamics of soft active matter. Rev Mod Phys 85: 1143-1189.

[11] Grégoire G, Chaté H (2004) Onset of Collective and Cohesive Motion. Phys Rev Lett 92:025702.

[12] Vicsek T, Zafeiris A (2012) Collective Motion. Phys Rep 517:71.

[13] Lau AWC, Lubensky TC (2009) Fluctuating hydrodynamics and microrheology of a dilute suspension of swimming bacteria. Phys. Rev. E 80:011917.

[14] Bialké J, Löwen H, Speck T (2013) Microscopic theory for the phase separation of self-propelled repulsive disks. EPL 103:30008.

[15] Thutupalli S, Seemann R, Herminghaus S (2011) Simple model squirmers with tunable velocity.

[16] Yamada D, Hondou T, Sano M (2003) Coherent dynamics of an asymmetric particle in a vertically vibrating bed. Phys. Rev. E 67: 040301.

[17] Dreyfus R et al. (2005) Microscopic Artificial Swimmers. Nature 437: 862.

[18] Keller EF, Segel LA (1971) Travelling bands of chemotactic bacteria: A theoretical analysis. $J$ Theor Biol 30:235.

[19] Paxton WF, et al. (2004) Catalytic Nanomotors: Autonomous Movement of Striped Nanorods. J Am Chem Soc 126:13424.

[20] Golestanian R, Liverpool TB, Ajdari A (2005) Propulsion of a Molecular Machine by Asymmetric Distribution of Reaction Products. Phys Rev Lett 94:220801.

[21] Howse JR, et al. (2007) Self-Motile Colloidal Particles: From Directed Propulsion to Random Walk. Phys Rev Lett 99:048102.

[22] Jiang, H-R, Yoshinaga N, Sano M (2010) Active Motion of a Janus Particle by Self- 
Thermophoresis in a Defocused Laser Beam. Phys Rev Lett 105:268302.

[23] Anderson JL (1989) Colloid transport by interfacial forces. Annu Rev Fluid Mech 21:61.

[24] Rückner G, Kapral R (2007) Chemically Powered Nanodimers. Phys Rev Lett 98:150603.

[25] Golestanian R, Liverpool TB, Ajdari A (2007) Designing phoretic micro- and nano-swimmers. New J Phys 9:126.

[26] Mitchell P (1956) Hypothetical thermokinetic and electrokinetic mechanisms of locomotion in micro-organisms. Proc. R. phys. Soc. Edinb. 25:32-34.

[27] Mitchell P (1972) Self-electrophoretic locomotion in microorganisms: bacterial flagella as giant ionophores. FEBS Lett. 28:1-5.

[28] Lammert PE, Prost J, Bruinsma R (1996) Ion drive for vesicles and cells. J. Theor. Biol. 178:387-391.

[29] Hong Y, Blackman NMK, Kopp ND, Sen A, Velegol D (2007) Chemotaxis of Non-Biological Nanorods. Phys. Rev. Lett. 99:178103.

[30] Ibele ME, Mallouk TE, Sen A (2009) Schooling Behavior of Light-Powered Autonomous Micromotors in Water. Angew. Chem. Int. Ed. 48:3308-3312.

[31] Golestanian R (2009) Anomalous Diffusion of Symmetric and Asymmetric Active Colloids. Phys. Rev. Lett. 102: 188305.

[32] Ibele ME et al. (2010) Emergent, Collective Oscillations of Self-Mobile Particles and Patterned Surfaces under Redox Conditions. ACS Nano 8:4845-4851.

[33] Kagan D, Balasubramanian S, Wang J (2011) Chemically Triggered Swarming of Gold Microparticles. Angew. Chem. Int. Ed. 50:503-506.

[34] Theurkauff I, Cottin-Bizonne C, Palacci J, Ybert C, Bocquet L (2012) Dynamic Clustering in Active Colloidal Suspensions with Chemical Signaling. Phys Rev Lett 108:268303.

[35] Palacci J, Sacanna S, Steinberg AP, Pine DJ, Chaikin PM (2013) Living Crystals of LightActivated Colloidal Surfers. Science 339:936-940.

[36] Duan W, Liu R, Sen A (2013) Transition between Collective Behaviors of Micromotors in Response to Different Stimuli. J. Am. Chem. Soc. 135:1280-1283.

[37] Baraban L, Harazim SM, Sanchez S, Schmidt OG (2013) Chemotactic Behavior of Catalytic Motors in Microfluidic Channels. Angew. Chem. Int. Ed. 52:5552-5556.

[38] Ebbens S, Tu MH, Howse JR, Golestanian R (2012) Size Dependence of the Propulsion Velocity for Catalytic Janus-Sphere Swimmers. Phys Rev E 85:020401.

[39] Cates M E (2012) Diffusive transport without detailed balance in motile bacteria: Does microbiology need statistical physics?. Rep Prog Phys 75:042601

[40] Nelson PC (2007) Biological Physics. WH Freeman

[41] Fair MC, Anderson JL (1989) Electrophoresis of nonuniformly charged ellipsoidal particles. J. Colloid Interface Sci. 127: 388.

[42] Alexander GP and Liu AP (2011) Self-Diffusiophoresis in the Advection Dominated Regime. arXiv:1107.3851.

[43] Jeans J (1929) Astronomy and Cosmogony, 2nd Edition. Cambridge Univ. Press, Cambridge.

[44] Lushi E, Goldstein RE, Shelley MJ (2012) Collective Chemotactic Dynamics in the Presence of Self-Generated Fluid Flows. Phys. Rev. E 86:040902.

[45] Zöttl A, Stark H (2013) Hydrodynamics determines collective motion and phase behavior of active colloids. arXiv:1309.4352,

[46] Lauga E, Thomas RP (2009) The hydrodynamics of swimming microorganisms. Rep. Prog. Phys. 72:096601.

[47] Bertin E, Droz M, Gregoire G (2006) Boltzmann and hydrodynamic description for self- 
propelled particles. Phy Rev E 74: 022101.

[48] Saintillan D, Shelley MJ (2008) Instabilities, pattern formation, and mixing in active suspensions Phys Fluids 20: 123304.

[49] Fily Y, Marchetti MC (2012) Athermal Phase Separation of Self-Propelled Particles with No Alignment. Phys Rev Lett 108: 235702.

[50] Gowrishankar K, Rao M (2012) Nonequilibrium phase transitions in active contractile polar filaments. arXiv:1201.3938v1.

[51] Golestanian R (2012) Collective Behavior of Thermally Active Colloids. Phys Rev Lett 108:038303.

[52] Tonks L, Langmuir I (1929) Oscillations in Ionized Gases. Phys. Rev. 33:195-210.

[53] For consistency with the nomenclature of enzyme catalysis literature we refer to the reactant as 'substrate', not to be confused with other uses of the term.

[54] A related idea has been investigated in the context of an ionic cell-motility mechanism [26 28].

[55] When this work was being prepared for submission, we learned of unpublished results from the groups of H Stark and J Brady on self-phoretic swimmers interacting through their diffusion fields. The construction of chemotactic behavior from the patterning of the colloid, the role of enzyme kinetics, the dynamics of orientation fields and aster formation, the occurrence of underdamped modes and the possibility of spontaneous oscillation are among the distinguishing features of our work.

[56] We will not discuss similar phoretic propulsion mechanisms through gradients in temperature or electrostatic potentials.

[57] Results from another limit of interest, $D_{p} \gg D_{s}$ and $s_{b} \ll \kappa_{1} / \kappa_{2}$, in which the chemical reaction influences significantly the local value of $s$ so that the coupled dynamics of orientation and translation can lead to oscillations, will be discussed elsewhere.

[58] We assume prompt replenishment of consumed substrate, and we work on timescales long enough that the product has reached the sample boundaries, where it is absorbed.

[59] In Eq. 3.1 we have displayed only those nonlinear terms required to stabilized a state of nonzero $\mathbf{n}_{L}$. The complete equations may be seen in the Supplementary Material.

\section{APPENDIX I: EXPRESSION FOR THE SOURCES}

We begin by obtaining an expression for the sources $Q(\mathbf{r}, t)$ for generation of $\mathrm{P}$ and consumption of $\mathrm{S}$ in terms of the coarse-grained density and orientation fields, $\sum_{\alpha} \delta\left(\mathbf{r}-\mathbf{r}_{\alpha}\right)=$ $\rho(\mathbf{r}), \sum_{\alpha} \hat{\mathbf{n}}_{\alpha} \delta\left(\mathbf{r}-\mathbf{r}_{\alpha}\right)=\mathbf{w}(\mathbf{r})$, of the colloids. $Q$ simply sums over all the enzymatic sites on the surfaces of all catalytic colloids. Setting $\mathbf{X}_{\alpha}=R \hat{\mathbf{x}}_{\alpha}$ (see Fig. VI), $\Omega_{b}$ as the infinitesimal 


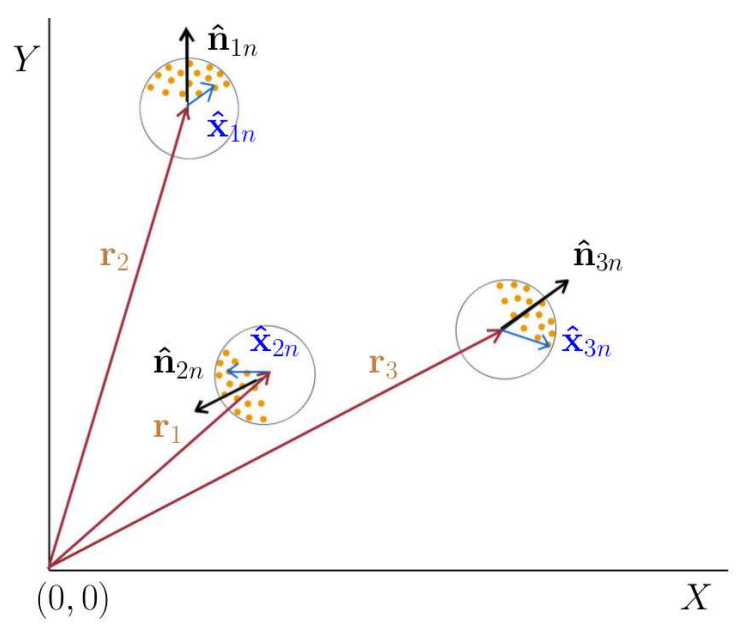

FIG. 5: Schematic for the calculation of $Q$.

solid angle and Taylor expanding upto $O\left(R^{3}\right)$ we get

$$
\begin{aligned}
Q(\mathbf{r}, t)= & \kappa(s) \sum_{\alpha} \int_{\left|\mathbf{X}_{\alpha}\right|=R} \delta\left(\mathbf{r}-\mathbf{r}_{\alpha}-\mathbf{X}_{\alpha}\right) \sigma\left(\mathbf{X}_{\alpha} \cdot \hat{\mathbf{n}}_{\alpha}\right) \\
= & \kappa(s) \sum_{\alpha} \int_{b} R^{2} \mathrm{~d} \Omega_{b}\left[\delta\left(\mathbf{r}-\mathbf{r}_{\alpha}\right)-R \hat{\mathbf{x}}_{\alpha} \cdot \nabla \delta\left(\mathbf{r}-\mathbf{r}_{\alpha}\right)+\frac{R^{2}}{2}\left(\hat{\mathbf{x}}_{\alpha} \cdot \nabla\right)^{2} \delta\left(\mathbf{r}-\mathbf{r}_{\alpha}\right)\right] \\
& \times\left[\sigma_{0}+\sigma_{1} \hat{\mathbf{x}}_{\alpha} \cdot \hat{\mathbf{n}}_{\alpha}+\sigma_{2}\left(\hat{\mathbf{x}}_{\alpha} \cdot \hat{\mathbf{n}}_{\alpha}\right)^{2}\right] \\
= & \kappa(s) 4 \pi R^{2}\left[\sigma_{0} \rho-\frac{1}{3} \sigma_{2} \rho-\frac{R \sigma_{1}}{3} \nabla \cdot \mathbf{n}\right] \\
= & \kappa(s) N\left[\rho-\frac{\sigma_{2}}{3 \sigma_{0}} \rho-\frac{R \sigma_{1}}{3 \sigma_{0}} \nabla \cdot \mathbf{n}\right]
\end{aligned}
$$

where $N=4 \pi R^{2} \sigma_{0}$. The sources are obtained using

$$
\int \hat{x}_{i} \hat{x}_{j} \mathrm{~d} \Omega_{b}=\frac{4 \pi}{3} \delta_{i j}
$$

\section{APPENDIX II: DERIVATION OF THE FULL NONLINEAR EQUATIONS}

Next, starting from the Langevin equations, we construct equations of motion for $\rho(\mathbf{r})$ and $\mathbf{w}(\mathbf{r})$. We construct the joint probability density $P$ for the position and orientation;

$$
P(\mathbf{r}, \mathbf{w})=\left\langle\sum_{\alpha} \delta\left(\mathbf{r}-\mathbf{r}_{\alpha}\right) \delta\left(\mathbf{w}-\hat{\mathbf{w}}_{\alpha}\right)\right\rangle
$$

$P$ is used to derive the Fokker Planck equation for the joint distribution of particle position and polarity;

$$
\begin{aligned}
& \partial_{t} P+\nabla \cdot\left[P\left(V_{0}(s) \hat{\mathbf{w}}+\alpha_{0} \nabla s+\alpha_{1} \hat{\mathbf{w}} \hat{\mathbf{w}} \cdot \nabla s+\beta_{0} \nabla p+\beta_{1} \hat{\mathbf{w}} \hat{\mathbf{w}} \cdot \nabla p\right)\right]-D \nabla^{2} P \\
& +\left(\hat{\mathbf{w}} \times \nabla_{w}\right) \cdot\left[P\left(\Phi_{0}(\hat{\mathbf{w}} \times \nabla s) \times \hat{\mathbf{w}}+\Omega_{0}(\hat{\mathbf{w}} \times \nabla p) \times \hat{\mathbf{w}}\right)\right]-D_{r}\left(\hat{\mathbf{w}} \times \nabla_{\mathbf{w}}\right)^{2} P=0(7.2)
\end{aligned}
$$


The equation for $\rho=\int P(\mathbf{r}, \hat{\mathbf{w}}) d^{2} w$ is given by taking the zeroth moment of the above equations

$\partial_{t} \rho+\nabla \cdot\left(V_{0} \mathbf{w}\right)+\nabla \cdot\left[\left(\beta_{0}+\frac{\beta_{1}}{3}\right) \nabla p-\left(\alpha_{0}+\frac{\alpha_{1}}{3}\right) \nabla s \rho+\left(\beta_{1} \nabla p-\alpha_{1} \nabla s\right) \cdot \mathbf{Q}\right]-D \nabla^{2} \rho=\left(\left(\nabla_{,}, 3\right)\right.$

where $D$ is the diffusivity of the swimmers and $\mathbf{Q}=\int P(\mathbf{r}, \hat{\mathbf{w}})(\hat{\mathbf{w}} \hat{\mathbf{w}}-\mathbf{I} / 3) d^{2} w$ is the nematic order parameter. The equation for the polar order parameter $\mathbf{w}(\mathbf{r})=\int P(\mathbf{r}, \hat{\mathbf{w}}) \hat{\mathbf{w}} d^{2} w$ is the first moment of (7.2)

$$
\begin{aligned}
\partial_{t} \mathbf{w} & +\nabla \cdot\left(V_{0} \mathbf{Q}\right)+\frac{1}{3} \nabla\left(V_{0} \rho\right)+\nabla \cdot\left[\left(-\alpha_{0} \nabla s+\beta_{0} \nabla p\right) \mathbf{w}+\left(-\alpha_{1} \nabla s+\beta_{1} \nabla p\right) \cdot \mathbf{Q}^{3}\right]-D \nabla^{2} \mathbf{w} \\
& +\Phi_{0}\left[\mathbf{Q} \cdot \nabla s-\frac{2}{3} \rho \nabla s\right]+\Omega_{0}\left[\mathbf{Q} \cdot \nabla p-\frac{2}{3} \rho \nabla p\right]+2 D_{r} \mathbf{w}=0
\end{aligned}
$$

where $\mathbf{Q}^{3}=\int P(\mathbf{r}, \mathbf{w}) \hat{\mathbf{w}}^{3} d^{2} w$. While coarse-graining, we see inevitably that the dynamical equation for $\mathbf{w}$ involves higher moments of the orientational distribution function which must be re-expressed in terms of lower moments [47? , 48]. No process involving apolar bundling of particles is considered here, so we do not consider strict nematic order. The occurrence of more than one factor of $\hat{\mathbf{w}}_{\alpha}$ can then lead only to higher powers of $\mathbf{w}$, combined with isotropic terms. We obtain a hierarchy of equation which have to be truncated using a closure condition.

\section{A. Closure}

The condition often used is to assume that $\mathbf{Q}$ relaxes fast so that $\partial_{t} \mathbf{Q}=0$, i.e. $\mathbf{Q}$ is slaved to $\rho$ and $\boldsymbol{w}$. In this scheme of closure we systematically ignore terms higher than $|\mathbf{n}|^{3}$ so that it holds close to the order disorder transition. Deep in the ordered state the closure scheme has to be modified keeping in mind that $\langle\mathbf{n n}\rangle=w_{0}^{2} \hat{\mathbf{k}} \hat{\mathbf{k}}$, where $\hat{\mathbf{k}}$ is the ordering direction and $w_{0}$ is magnitude, so that one has to look at fluctuations about this reference state.

We will now write down the equation of motion for the nematic order parameter and obtain an expression for $\mathbf{Q}$ in terms of $\rho$ and $\mathbf{n}$. The expression for $\mathbf{Q}$ is substituted in the equations for $\rho$ and $\mathbf{n}$ to obtain coarse grained equations of these variables in terms of $\rho$ and $\mathbf{n}$ and involving the diffusion field $s$. Next we work in two different approximations to obtain expressions for $s$ in terms of $\rho$ and $\mathbf{n}$ to obtain effective equations for $\rho$ and $\mathbf{n}$. The nematic order parameter $\mathbf{Q}$ satisfies the equation

$$
\begin{aligned}
& \partial_{t} \mathbf{Q}+\nabla \cdot\left[V_{0}\left(\mathbf{Q}^{3}-\frac{\mathbf{I}}{3} \mathbf{w}\right)\right]-\alpha_{0} \nabla \cdot(\nabla s \mathbf{Q})-\alpha_{1} \nabla \cdot\left(\mathbf{Q}^{4} \cdot \nabla s-\frac{\mathbf{I}}{3} \mathbf{Q}^{\prime} \cdot \nabla s\right)+\beta_{0} \nabla \cdot(\nabla p \mathbf{Q}) \\
& +\beta_{1} \nabla \cdot\left(\mathbf{Q}^{4} \cdot \nabla p-\frac{\mathbf{I}}{3} \mathbf{Q}^{\prime} \cdot \nabla p\right)-D \nabla^{2} \mathbf{Q}+2 \Phi_{0}\left(\mathbf{Q}^{3} \cdot \nabla s-\mathbf{w} \nabla s\right)+2 \Omega_{0}\left(\mathbf{Q}^{3} \cdot \nabla p-\mathbf{w} \nabla p\right) \\
& +6 D_{r} \mathbf{Q}=0
\end{aligned}
$$

where $\mathbf{Q}^{4}=\int P(\mathbf{r}, \mathbf{w}) \hat{\mathbf{w}}^{4} d^{2} w$ and $\mathbf{Q}^{\prime}=\int P(\mathbf{r}, \mathbf{w}) \hat{\mathbf{w}}^{2} d^{2} w$.

The traceless parts of higher moments like $\mathbf{Q}^{3}$ and $\mathbf{Q}^{4}$ are assumed to be negligibly small, so that

$$
\begin{aligned}
Q_{\alpha \beta \gamma}^{3} & \rightarrow \frac{1}{5}\left(w_{\alpha} \delta_{\beta \gamma}+w_{\beta} \delta_{\gamma \alpha}+w_{\gamma} \delta_{\alpha \beta}\right) \\
Q_{\alpha \beta \gamma \mu}^{4} & \rightarrow \frac{1}{15}\left(\delta_{\alpha \beta} \delta_{\gamma \mu}+\delta_{\alpha \gamma} \delta_{\beta \mu}+\delta_{\alpha \mu} \delta_{\gamma \beta}\right)
\end{aligned}
$$


Using (7.6) and $p=-s D_{s} / D_{p}$, and defining for any two vectors $\mathbf{v}_{1}$ and $\mathbf{v}_{2}$,

$$
\left(\mathbf{v}_{1} \mathbf{v}_{2}\right)^{T S}=\frac{1}{2}\left(\mathbf{v}_{1} \mathbf{v}_{2}+\mathbf{v}_{2} \mathbf{v}_{1}\right)-\left(\mathbf{v}_{1} \cdot \mathbf{v}_{2}\right) \frac{\mathbf{l}}{3},
$$

and keeping terms upto the lowest order in gradients we have

$$
\mathbf{Q}=\frac{1}{15 D_{r}}\left[-\mathbf{n} \nabla V_{0}-V_{0} \nabla \mathbf{n}+\frac{C_{3}}{3} \rho \nabla \nabla s+\frac{C_{3}}{3} \nabla \rho \nabla s-3 C_{2} \mathbf{n} \nabla s\right]^{T S},
$$

where

$$
\begin{aligned}
C_{1} & =\alpha_{0}+\frac{\alpha_{1}}{5}+\frac{D_{s}}{D_{p}}\left(\beta_{0}+\frac{\beta_{1}}{5}\right) \\
C_{2} & =\frac{D_{s}}{D_{p}} \Omega_{0}-\Phi_{0} \\
C_{3} & =\alpha_{1}+\frac{D_{s}}{D_{p}} \beta_{1} .
\end{aligned}
$$

We also define

$$
\begin{aligned}
B & =N \kappa(s)\left[\frac{1}{D_{s}}\left(\alpha_{0}+\frac{\alpha_{1}}{3}\right)+\frac{1}{D_{p}}\left(\beta_{0}+\frac{\beta_{1}}{3}\right)\right], \\
A & =N \kappa(s)\left[\frac{\Phi_{0}}{D_{s}}-\frac{\Omega_{0}}{D_{p}}+\Delta \frac{V_{0}\left(s_{0}\right)}{2 D_{s}}\right]
\end{aligned}
$$

where $\Delta=d \ln \kappa(s) /\left.d s\right|_{s_{0}}$.

\section{B. Saturated: Equation of Motion for $\mathbf{n}$}

In this regime, the phoretic velocity is independent of the substrate concentration so that $\nabla V_{0}=0$ and we substitute $\lim _{s_{0}>>\kappa_{2} / \kappa_{1}} V_{0}(s) \rightarrow v_{0}$ and $\lim _{s_{0}>>\kappa_{2} / \kappa_{1}} \kappa\left(s_{0}\right) \rightarrow \kappa_{2}$. Substituting $\mathbf{Q}$ from (7.8) into (7.4) and using

$$
\mathbf{Q}^{3} \cdot \nabla s=\frac{1}{5}(\mathbf{n} \nabla s+\nabla s \mathbf{n}+(\mathbf{n} \cdot \nabla s) \mathbf{I})
$$

we have the equation of motion for the polar order parameter

$$
\begin{aligned}
& \partial_{t} \mathbf{n}-\left(D+\frac{v_{0}^{2}}{30 D_{r}}\right) \nabla^{2} \mathbf{n}-\frac{v_{0}^{2}}{90 D_{r}} \nabla(\nabla \cdot \mathbf{n})+2 D_{r} \mathbf{n}+\frac{2 C_{2}}{3} \rho \nabla s+\frac{v_{0}}{3} \nabla \rho \\
& -\left(\frac{11 C_{2} v_{0}}{90 D_{r}}+\frac{C_{3}}{5}\right)(\nabla \cdot \mathbf{n}) \nabla s-\left(\frac{C_{2} v_{0}}{10 D_{r}}+\frac{C_{3}}{5}\right)(\mathbf{n} \cdot \nabla) \nabla s-\left(\frac{C_{2} v_{0}}{10 D_{r}}+C_{1}\right)\left(\nabla^{2} s\right) \mathbf{n} \\
& -\left(\frac{C_{2} v_{0}}{15 D_{r}}+C_{1}\right)(\nabla s \cdot \nabla) \mathbf{n}-\left(\frac{C_{3}}{5}-\frac{C_{2} v_{0}}{15 D_{r}}\right) \nabla(\mathbf{n} \cdot \nabla s)+\frac{C_{2} v_{0}}{30 D_{r}}(\nabla \mathbf{n}) \cdot \nabla s \\
& +\frac{C_{3} v_{0}}{270 D_{r}}\left[4 \rho \nabla\left(\nabla^{2} s\right)+3(\nabla s \cdot \nabla) \nabla \rho-2 \nabla(\nabla \rho \cdot \nabla s)+9(\nabla \rho \cdot \nabla) \nabla s+3 \nabla^{2} \rho \nabla s+\left(\nabla^{2} s\right) \nabla \rho\right] \\
& +\frac{C_{2}^{2}}{10 D_{r}} \mathbf{n}|\nabla s|^{2}+\frac{C_{2}^{2}}{30 D_{r}}(\mathbf{n} \cdot \nabla s) \nabla s \\
& +\frac{C_{2} C_{3}}{270 D_{r}}\left[2 \rho\left(\nabla^{2} s\right) \nabla s-6 \rho(\nabla \nabla s) \cdot \nabla s-3 \nabla \rho|\nabla s|^{2}-\nabla s(\nabla s \cdot \nabla \rho)\right]=0
\end{aligned}
$$


In the saturated limit we have $\nabla s=N \kappa_{2}\left(\boldsymbol{E}-\epsilon \mathbf{w}_{L}\right) / D_{s}$ and $\nabla^{2} s=N \kappa_{2}(\rho-\epsilon \nabla \cdot \mathbf{w}) / D_{s}$. We have defined the curl free or longitudinal component of $\mathbf{n}$ expressed in the fourier space as $\mathbf{n}_{\mathbf{q} L}=\hat{\mathbf{q}} \hat{\mathbf{q}} \cdot \mathbf{n}_{\mathbf{q}}$. In what follows we use $p=-s D_{s} / D_{p}$ and define $c_{1,3}=N \kappa_{2} C_{1,3} / D_{s}$. The effective equation for $\mathbf{w}$ is then

$$
\begin{aligned}
& \partial_{t} \mathbf{n}-\left(D+\frac{v_{0}^{2}}{30 D_{r}}\right) \nabla^{2} \mathbf{n}+\left(\frac{2 \epsilon c_{3} v_{0}}{135 D_{r}} \rho-\frac{v_{0}^{2}}{90 D_{r}}\right) \nabla(\nabla \cdot \mathbf{n})+\left(\frac{v_{0}}{3}+f_{1}\right) \nabla \rho \\
& +\left(2 D_{r}+f_{2}\right) \mathbf{n}+\left(\frac{A^{2} \epsilon^{2}}{30 D_{r}}\left|\mathbf{n}_{L}\right|^{2}-\frac{2 A \epsilon}{3} \rho+f_{3}\right) \mathbf{n}_{L}+\left(\frac{2 A}{3} \rho-f_{4}\right) \mathbf{E} \\
& +\nabla \Pi+[\mathbf{n} \nabla \mathbf{E}]+[\mathbf{n n E}]+\left[\left(\mathbf{E}-\epsilon \mathbf{n}_{L}\right) \nabla \rho\right]+[\mathbf{n} \nabla \mathbf{n}]=0,
\end{aligned}
$$

where the square brackets denote a linear combination of all possible contractions of the enclosed terms to obtain a vector. The equation is asymptotically exact as $\xi_{s} \rightarrow \infty$. We will now discuss the significance of various terms and provide expressions for the functions introduced above. As the swimmer moves with an average speed $v_{0}$ along its symmetry axis, the polar order parameter is equivalent to a velocity field. With this interpretation $\rho$ becomes a pressure like term where corrections to the squared sound speed $v_{0} / 3$ is given by

$$
f_{1}=-\frac{v_{0} c_{3}}{270 D_{r}} \delta \rho-\frac{\epsilon v_{0} c_{3}}{90 D_{r}}\left(\nabla \cdot \mathbf{n}_{L}\right)
$$

Similarly $\Pi$ is a generalised pressure term depending on the other fields as

$$
\Pi=\left(\mathbf{E}-\epsilon \mathbf{n}_{L}\right) \cdot\left[\left(\frac{v_{0} A}{15 D_{r}}-\frac{c_{3}}{5}+\frac{\epsilon A c_{3}}{90 D_{r}} \rho\right) \mathbf{n}_{L}-\frac{v_{0} c_{3}}{135 D_{r}} \nabla \rho-\frac{A c_{3}}{90 D_{r}} \rho \mathbf{E}\right] .
$$

The presence of the swimmer creates spatial variation in the $\mathrm{S}$ and $\mathrm{P}$. This is encoded in the fields $\mathbf{E}$ and $\mathbf{n}_{L}$ of which the first enters (7.13) as an external orienting field while the second enters the dynamics as a spontaneous symmetry breaking effect where the linear term $\left(D_{r}-2 \rho A / 3\right)$ can change sign producing $\mathbf{n}_{L}$ condensation. There are higher order stabilising terms with coefficient $\propto A^{2}$ which restrain the growth of $\left|\mathbf{n}_{L}\right|$. The linear terms coefficients are modified by the corrections

$$
\begin{aligned}
& f_{2}=\frac{\epsilon^{2} A^{2}}{10 D_{r}}\left|\mathbf{n}_{L}\right|^{2}+\delta \rho\left(c_{1}+\frac{A v_{0}}{10 D_{r}}\right)+\frac{A^{2}}{10 D_{r}}|\mathbf{E}|^{2}, \\
& f_{3}=\frac{\epsilon A c_{3}}{45 D_{r}}\left[\frac{1}{6} \nabla \rho \cdot\left(\mathbf{E}-\epsilon \mathbf{n}_{L}\right)-\rho \delta \rho\right], \\
& f_{4}=\frac{A c_{3}}{135 D_{r}} \rho \delta \rho+\frac{\epsilon^{2} A^{2}}{30 D_{r}}\left|\mathbf{n}_{L}\right|^{2}+\frac{A c_{3}}{270 D_{r}} \nabla \rho \cdot\left(\mathbf{E}-\epsilon \mathbf{n}_{L}\right),
\end{aligned}
$$

which can destabilise the condensed state. Note that the terms that dictate the nature of the aster condensation are all proportional to the polarity of the catalytic coat as they are sensitive to the polar nature of the diffusion field.

The advective nonlinearities have the same structure as in the Toner-Tu model [8] given that $\mathbf{n}$ is like a velocity that can carry other fields. However note the absence of terms of the form $(\mathbf{n} \cdot \nabla) \mathbf{n}$; this is because the interaction in the two cases are very different and in this particular closure that holds close to the transition the average value of $\mathbf{n}$ and thus the coefficients of advective terms are proportional to the interaction strength which in this case 
is $\epsilon$. Bertin et. al. [47] modelled the Toner-Tu like interaction as an actual binary collision which tends to align the particles. In this system where interactions are mediated by the diffusion fields alone, the interaction is mediated by the $\mathbf{n}_{L}$ term as discussed above.

$$
\begin{aligned}
{[\mathbf{n} \nabla \mathbf{n}] } & \equiv \epsilon\left(\frac{c_{3}}{5}-\frac{A c_{3} \epsilon}{135 D_{r}} \rho+\frac{11 A v_{0}}{90 D_{r}}\right)(\nabla \cdot \mathbf{n}) \mathbf{n}_{L}+\epsilon\left(\frac{A v_{0}}{10 D_{r}}+\frac{c_{3}}{5}\right)(\mathbf{n} \cdot \nabla) \mathbf{n}_{L} \\
-\epsilon & \left(\frac{A v_{0}}{10 D_{r}}+c_{1}\right) \mathbf{n}(\nabla \cdot \mathbf{n})+\epsilon\left(\frac{A v_{0}}{15 D_{r}}+c_{1}\right)\left(\mathbf{n}_{L} \cdot \nabla\right) \mathbf{n}-\epsilon \frac{A v_{0}}{30 D_{r}} \mathbf{n}_{L} \times(\nabla \times \mathbf{n}) .
\end{aligned}
$$

The other nonlinearities are the terms coupling $\mathbf{n}$ and $\mathbf{E}$ which to lowest order are

$$
\begin{aligned}
{[\mathbf{n} \nabla \mathbf{E}] \equiv } & \left(\frac{c_{3}}{5}-\frac{A c_{3} \epsilon}{135 D_{r}} \rho+\frac{11 A v_{0}}{90 D_{r}}\right)(\nabla \cdot \mathbf{n}) \mathbf{E}+\left(c_{1}+\frac{A v_{0}}{15 D_{r}}\right)(\mathbf{E} \cdot \nabla) \mathbf{n} \\
& +\left(\frac{c_{3}}{5}+\frac{A v_{0}}{10 D_{r}}\right)(\mathbf{n} \cdot \nabla) \mathbf{E}+\frac{A v_{0}}{30 D_{r}} \mathbf{E} \times(\nabla \times \mathbf{n}),
\end{aligned}
$$

and higher order terms

$$
[\mathbf{n n E}] \equiv-\frac{\epsilon A^{2}}{45 D_{r}} \mathbf{n}\left(\mathbf{n}_{L} \cdot \mathbf{E}\right)-\frac{\epsilon A^{2}}{30 D_{r}} \mathbf{n}_{L}(\mathbf{n} \cdot \mathbf{E})+\frac{A^{2}}{30 D_{r}}(\mathbf{n} \cdot \mathbf{E}) \mathbf{E}
$$

The rest of the nonlinearities couple $\nabla \rho$ and $\left(\mathbf{E}-\epsilon \mathbf{n}_{L}\right)$ :

$\left[\nabla \rho\left(\mathbf{E}-\epsilon \mathbf{n}_{L}\right)\right]=\frac{v_{0} c_{3}}{90 D_{r}}\left[\left(\mathbf{E}-\epsilon \mathbf{n}_{L}\right) \cdot \nabla\right] \nabla \rho+\frac{v_{0} c_{3}}{30 D_{r}}(\nabla \rho \cdot \nabla)\left(\mathbf{E}-\epsilon \mathbf{n}_{L}\right)+\frac{v_{0} c_{3}}{90 D_{r}}\left(\mathbf{E}-\epsilon \mathbf{n}_{L}\right) \nabla^{2} \rho$.

We linearise (7.13) to obtain

$$
\begin{aligned}
& \partial_{t} \mathbf{n}-\left(D+\frac{v_{0}^{2}}{30 D_{r}}\right) \nabla^{2} \mathbf{n}+\left(\frac{2 \epsilon c_{3} v_{0} \rho_{0}}{135 D_{r}}-\frac{v_{0}^{2}}{90 D_{r}}\right) \nabla(\nabla \cdot \mathbf{n})+\left(\frac{v_{0}}{3}-\frac{2 c_{3} v_{0} \rho_{0}}{135 D_{r}}\right) \nabla \rho+2 D_{r} \mathbf{n}-\frac{2 A \epsilon}{3} \rho_{0} \mathbf{n}_{L} \\
& +\frac{2 A}{3} \rho_{0} \mathbf{E}=0
\end{aligned}
$$

Defining $\mathbf{n}$ by $\mathbf{n} / \rho_{0}$; and the transverse component of $\mathbf{n}$ as $\mathbf{n}_{\mathbf{q} t}=(\mathbf{I}-\hat{\mathbf{q}} \hat{\mathbf{q}}) \cdot \mathbf{n}_{\mathbf{q}}$ we get

$$
\begin{aligned}
& {\left[\partial_{t}-\left(D+\frac{v_{0}^{2}}{30 D_{r}}\right) \nabla^{2}-\left(\frac{v_{0}^{2}}{90 D_{r}}-\frac{2 \epsilon c_{3} v_{0} \rho_{0}}{135 D_{r}}\right) \nabla \nabla \cdot\right] \mathbf{n}_{L}+\left(\frac{4 \epsilon^{2} A^{2}}{30 D_{r}}+2 D_{r}-\frac{2 A \epsilon \rho_{0}}{3}\right) \mathbf{n}_{L}} \\
& +\rho_{0}^{-1}\left(\frac{v_{0}}{3}-\frac{2 c_{3} v_{0} \rho_{0}}{135 D_{r}}\right) \nabla \rho+\frac{2 A}{3} \mathbf{E}=0 \\
& {\left[\partial_{t}-\left(D+\frac{v_{0}^{2}}{30 D_{r}}\right) \nabla^{2}+\left(\frac{2 \epsilon c_{3} v_{0} \rho_{0}}{135 D_{r}}-\frac{v_{0}^{2}}{90 D_{r}}\right) \nabla \nabla \cdot 16\right)}
\end{aligned}
$$

Note that for $D_{r}>0, \mathbf{n}_{t}$ stays in the overdamped region and is a fast degree of freedom that we throw away. Fourier transforming Eq.(17.16) we get

$$
\left.\left[\partial_{t}+\left(D+\frac{v_{0}^{2}}{90 D_{r}}-\frac{2 \epsilon N \kappa_{2} v_{0} \rho_{0}}{135 D_{r}}\left(\frac{\alpha_{1}}{D_{s}}+\frac{\beta_{1}}{D_{p}}\right)\right) q^{2}+2\left(D_{r}-\frac{\epsilon \rho_{0} A}{3}\right)\right] i \mathbf{q} \cdot \mathbf{n}_{l \mathbf{q}}-\frac{v_{0}}{3 \rho_{0}} q^{2} \rho_{\mathbf{q}}+\frac{2 A}{3} \rho_{l \mathbf{q}} \neq 7018\right)
$$




\section{Saturated: Equation of Motion for $\rho$} for $\rho$

Substituting the closed form for $\mathbf{Q}$ from Eq. (17.11) into Eq. (17.3) we get the equation

$$
\begin{aligned}
& \partial_{t} \rho+v_{0} \nabla \cdot \mathbf{n}-\frac{B D_{s}}{\kappa_{2} N} \nabla \cdot(\rho \nabla s)-D \nabla^{2} \rho \\
& -\frac{C_{3}}{15 D_{r}} \nabla \cdot\left[-\frac{v_{0}}{2}(\nabla \mathbf{n}) \cdot \nabla s-\frac{v_{0}}{2}(\nabla s \cdot \nabla) \mathbf{n}+\frac{v_{0}}{3}(\nabla \cdot \mathbf{n}) \nabla s-\frac{A}{2}(\nabla s \cdot \mathbf{n}) \nabla s\right. \\
& \left.+\frac{C_{3}}{3} \rho(\nabla \nabla s) \cdot \nabla s-\frac{C_{3}}{9} \rho(\nabla s) \nabla^{2} s+\frac{C_{3}}{3} \nabla \rho|\nabla s|^{2}+\frac{C_{3}}{6} \nabla \rho(\nabla s \cdot \nabla \rho)\right]=0
\end{aligned}
$$

Substituting for $\nabla s$ and using $c_{1,3}=N \kappa_{2} C_{1,3} D_{s}^{-1}$ we have

$$
\begin{aligned}
& \partial_{t} \rho+v_{0} \nabla \cdot \mathbf{n}-D \nabla^{2} \rho-\nabla \cdot\left[\mathbf{E}\left(B \rho+\frac{\epsilon c_{3} A}{30 D_{r}} w_{L}^{2}+\frac{c_{3}^{2}}{135 D_{r}} \rho \delta \rho\right)\right] \\
& +\epsilon \nabla \cdot \mathbf{n}_{L}\left(B \rho+\frac{\epsilon c_{3}^{2}}{135 D_{r}} \rho \delta \rho+\frac{\epsilon^{2} c_{3} A}{30 D_{r}} w_{L}^{2}\right)-\frac{c_{3}}{15 D_{r}} \nabla \cdot \mathcal{J}=0
\end{aligned}
$$

where $\delta \rho=\rho-\rho_{0}$ and $\mathcal{J}$ is the nonlinear part of the current:

$$
\begin{aligned}
& \mathcal{J}=\left(\frac{\epsilon c_{3}}{9} \rho-\frac{v_{0}}{3}\right)(\nabla \cdot \mathbf{n}) \mathbf{E}+v_{0}(\mathbf{E} \cdot \nabla) \mathbf{n}+\frac{v_{0}}{2} \mathbf{E} \times(\nabla \times \mathbf{n})+\frac{A}{2}(\mathbf{n} \cdot \mathbf{E}) \mathbf{E}-\frac{A \epsilon}{2}(\mathbf{n} \cdot \mathbf{E}) \mathbf{n}_{L} \\
& -\epsilon v_{0}\left(\mathbf{n}_{L} \cdot \nabla\right) \mathbf{n}+\left(\frac{\epsilon v_{0}}{3}-\frac{\epsilon^{2} c_{3}}{9} \rho\right)(\nabla \cdot \mathbf{n}) \mathbf{n}_{L}-\frac{v_{0} \epsilon}{2} \mathbf{n}_{L} \times(\nabla \times \mathbf{n}) \\
& +\frac{c_{3}}{3} \nabla \rho\left|\mathbf{E}-\epsilon \mathbf{n}_{L}\right|^{2}+\frac{c_{3} D_{s}}{6 N \kappa_{2}} \nabla \rho\left(\nabla \rho \cdot \mathbf{E}-\epsilon \nabla \rho \cdot \mathbf{n}_{L}\right)
\end{aligned}
$$

Eq (7.20) is linearised and written in terms of $\mathbf{n}$ :

$$
\left(\partial_{t}-D \nabla^{2}\right) \rho+\rho_{0}\left(v_{0}+\epsilon B \rho_{0}\right) \nabla \cdot \mathbf{n}_{L}-B \rho_{0} \nabla \cdot \mathbf{E}=0
$$

Fourier transforming we get

$$
\left(\partial_{t}+D q^{2}-B \rho_{0}\right) \rho_{\mathbf{q}}+\rho_{0}\left(v_{0}+\epsilon B \rho_{0}\right) i \mathbf{q} \cdot \mathbf{n}_{L \mathbf{q}}=0
$$

\section{Unsaturated: equation of motion for $\mathbf{n}$}

In the linear part of the MM curve where $\kappa(s)=\kappa_{1} s, V_{0}$ varies linearly with substrate concentration so that we can define the self phoretic velocity as $V_{0}(s)=v_{1} s$. The equation 
for $\mathbf{n}$ in the unsaturated limit is

$$
\begin{aligned}
& \partial_{t} \mathbf{n}-\left(D+\frac{v_{1}^{2} s^{2}}{30 D_{r}}\right) \nabla^{2} \mathbf{n}-\frac{v_{1}^{2} s^{2}}{90 D_{r}} \nabla(\nabla \cdot \mathbf{n})+\frac{2 C_{2}}{3} \rho \nabla s+\frac{v_{1}}{3} \nabla(s \rho)+2 D_{r} \mathbf{n} \\
& +\left[\frac{\left(v_{1}-11 C_{2}\right) v_{1}}{90 D_{r}} s-\frac{C_{3}}{5}\right](\nabla \cdot \mathbf{n}) \nabla s-\left[\frac{\left(C_{2}+5 v_{1}\right) v_{1}}{30 D_{r}} s+C_{1}\right](\nabla s \cdot \nabla) \mathbf{n}-\left[\frac{\left(3 C_{2}+v_{1}\right) v_{1}}{30 D_{r}} s+\frac{C_{3}}{5}\right](\mathbf{n} \cdot \\
& -\left[\frac{\left(3 C_{2}+v_{1}\right) v_{1}}{30 D_{r}} s+C_{1}\right] \mathbf{n} \nabla^{2} s+\left[\frac{\left(3 C_{2}+v_{1}\right) v_{1}}{45 D_{r}} s-\frac{C_{3}}{5}\right] \nabla(\mathbf{n} \cdot \nabla s)-\left[\frac{\left(2 v_{1}-C_{2}\right) v_{1}}{30 D_{r}}\right] \nabla s \times(\nabla \times \mathbf{n}) \\
& +\frac{2 C_{3} v_{1}}{135 D_{r}} s \nabla^{2}(\nabla s)-\frac{\left(v_{1}-C_{2}\right)\left(3 C_{2}+v_{1}\right)}{90 D_{r}}\left[\mathbf{n}|\nabla s|^{2}+3 \nabla s(\mathbf{n} \cdot \nabla s)\right] \\
& +\frac{C_{3}\left(v_{1}-C_{2}\right)}{90 D_{r}}\left[\rho \nabla|\nabla s|^{2}-\frac{2}{3} \rho \nabla^{2} s \nabla s\right]+\frac{\left(C_{2}+v_{1}\right) C_{3}}{90 D_{r}}\left[\frac{1}{3} \nabla s(\nabla s \cdot \nabla \rho)+\nabla \rho|\nabla s|^{2}\right] \\
& +\frac{C_{3} v_{1} s}{270 D_{r}}\left[9(\nabla \rho \cdot \nabla) \nabla s-2\left(\nabla^{2} s\right) \nabla \rho+\left(\nabla^{2} \rho\right) \nabla s+3(\nabla s \cdot \nabla) \nabla \rho-2 \nabla(\nabla \rho \cdot \nabla s)\right]=0
\end{aligned}
$$

To obtain the effective equation for $\mathbf{n}$ we substitute $s \rightarrow s_{0}+\delta s$; in the highly unsaturated limit we have $\delta s=-\xi_{s}^{2} N \kappa\left(s_{0}\right)(\delta \rho-\epsilon \nabla \cdot \mathbf{n}) / D_{s}$ for $q<<\xi_{s}^{-1}$. Keeping terms upto second order in gradients we get

$$
\begin{aligned}
& \partial_{t} \mathbf{n}-\left(D+\frac{v_{1}^{2} s_{0}^{2}}{30 D_{r}}-\frac{2 N \xi_{s}^{2} v_{1}^{2} s_{0} \kappa}{30 D_{r} D_{s}} \delta \rho\right) \nabla^{2} \mathbf{n}+\left(\frac{\xi_{s}^{2} A}{3} \rho-\frac{v_{1}^{2} s_{0}^{2}}{90 D_{r}}+\frac{2 N \kappa \xi_{s}^{2} v_{1}^{2} s_{0}}{90 D_{r}} \delta \rho\right) \nabla(\nabla \cdot \mathbf{n})+2 D_{r} \mathbf{n} \\
& +\nabla \rho\left[\frac{v_{1} s_{0}}{3}-\frac{\xi_{s}^{2} A}{3} \rho-\frac{N \kappa \xi_{s}^{2} v_{1}}{3}(\delta \rho-\epsilon \nabla \cdot \mathbf{n}) \nabla \rho\right] \\
& -\frac{\xi_{s}^{4}\left(3 v_{1} N \kappa-A D_{s}\right)\left(3 A D_{s}-v_{1} N \kappa\right)}{360 D_{r} D_{s}^{2}}\left[3 \mathbf{n}|\nabla \rho|^{2}+\nabla \rho(\mathbf{n} \cdot \nabla \rho)\right] \\
& +\xi_{s}^{2}[\nabla \rho \nabla \mathbf{n}]=0
\end{aligned}
$$

The pressure is modified due to two separate contributions: (1) because of chemotactic alignment of swimmers with the local chemical gradient through $\rho \nabla s$ and (2) due to the change in self phoretic velocity with change in background substrate concentration through the term $\nabla(s \rho)$. The advective nonlinearities are

$$
\begin{aligned}
& {[\nabla \rho \nabla \mathbf{n}]=} \\
& -\left[\frac{\left(13 v_{1} N \kappa-11 A D_{s}\right) v_{1} s_{0}}{180 D_{r} D_{s}}-\frac{N \kappa C_{3}}{5 D_{s}}\right](\nabla \cdot \mathbf{n}) \nabla \rho+\left[\frac{\left(A D_{s}+9 v_{1} N \kappa\right) v_{1} s_{0}}{60 D_{r} D_{s}}+\frac{N \kappa C_{1}}{D_{s}}\right](\nabla \rho \cdot \nabla) \mathbf{n} \\
& +\left[\frac{\left(3 A D_{s}-v_{1} N \kappa\right) v_{1} s_{0}}{60 D_{r} D_{s}}+\frac{C_{3} N \kappa}{5 D_{s}}\right](\mathbf{n} \cdot \nabla) \nabla \rho+\left[\frac{\left(3 A D_{s}-v_{1} N \kappa\right) v_{1} s_{0}}{60 D_{r} D_{s}}+\frac{N \kappa C_{1}}{D_{s}}\right] \mathbf{n} \nabla^{2} \rho \\
& +\left[\frac{C_{3} N \kappa}{5 D_{s}}-\frac{\left(3 A D_{s}-v_{1} N \kappa\right) v_{1} s_{0}}{90 D_{r} D_{s}}\right] \nabla(\mathbf{n} \cdot \nabla \rho)+\frac{\left(5 v_{1} N \kappa-A D_{s}\right) v_{1}}{60 D_{r} D_{s}} \nabla \rho \times(\nabla \times \mathbf{n}) .
\end{aligned}
$$

Note that the advective nonlinearities given in Eqs. (7.15) and (7.26) are very different in the two regimes because in the unsaturated limit $\mathbf{n}_{L}$ acts like an ordering field while in the other the local chemical gradient is given by $\nabla(\nabla \cdot \mathbf{n})$. The linearised equation for $\mathbf{n}$ is

$\partial_{t} \mathbf{n}-\left(D+\frac{v_{1}^{2} s_{0}^{2}}{30 D_{r}}\right) \nabla^{2} \mathbf{n}-\frac{v_{1}^{2} s_{0}^{2}}{90 D_{r}} \nabla(\nabla \cdot \mathbf{n})-A \xi_{s}^{2} \rho_{0} \nabla(\rho-\epsilon \nabla \cdot \mathbf{n})+\frac{v_{1} s_{0}}{3} \nabla \rho+2 D_{r} \mathbf{n}=\left(\mathrm{T}_{0} .27\right)$ 
The equation for $\mathbf{n}_{L}$ and $\mathbf{n}_{t}$ are

$$
\begin{aligned}
& {\left[\partial_{t}+2 D_{r}-\left(D+\frac{v_{1}^{2} s_{0}^{2}}{30 D_{r}}\right) \nabla^{2}-\frac{v_{1}^{2} s_{0}^{2}}{90 D_{r}} \nabla \nabla \cdot\right] \mathbf{n}_{L}+\frac{\epsilon \rho_{0} \xi_{s}^{2} A}{3} \nabla\left(\nabla \cdot \mathbf{n}_{L}\right)+\left[\frac{v_{1} s_{0}}{3 \rho_{0}}-\frac{\xi_{s}^{2} A}{3}\right] \nabla \rho=0} \\
& {\left[\partial_{t}+2 D_{r}-\left(D+\frac{v_{1}^{2} s_{0}^{2}}{30 D_{r}}\right) \nabla^{2}\right] \mathbf{n}_{t}=0}
\end{aligned}
$$

Fourier transforming the equation for $\mathbf{n}_{L}$ get

$$
\left[\partial_{t}+2 D_{r}+\left(D+\frac{2 v_{1}^{2} s_{0}^{2}}{45 D_{r}}\right) q^{2}\right] i \mathbf{q} \cdot \mathbf{n}_{L \mathbf{q}}-q^{2} \frac{\left[v_{1} s_{0}-\rho_{0} A \xi_{s}^{2}\right]}{3 \rho_{0}} \rho_{\mathbf{q}}-q^{2} \frac{\epsilon \rho_{0} A}{3} i \mathbf{q} \cdot \mathbf{n}_{L \mathbf{q}}=0
$$

\section{E. Unsaturated: equation of motion for $\rho$}

The equation for $\rho$ is

$$
\partial_{t} \rho-D \nabla^{2} \rho+v_{1} s(\nabla \cdot \mathbf{n})-\frac{B D_{s}}{N \kappa} \rho\left(\nabla^{2} s\right)-\frac{B D_{s}}{N \kappa}(\nabla s \cdot \nabla) \rho+\frac{C_{3}}{45 D_{r}} \nabla \cdot \mathcal{J}(\nabla s, \nabla \rho, \rho, s \gamma 7.30)
$$

where $\mathcal{J}$ is the nonlinear part of the current. Substituting for $\delta s$, the effective equation for $\rho$ upto $O\left(\nabla^{3}\right)$ is

$$
\begin{aligned}
& \partial_{t} \rho-\left(D-B \xi_{s}^{2} \rho\right) \nabla^{2} \rho-\epsilon B \xi_{s}^{2} \rho \nabla^{2}(\nabla \cdot \mathbf{n})+B \xi_{s}^{2}\left[|\nabla \rho|^{2}-\epsilon \nabla \rho \cdot \nabla(\nabla \cdot \mathbf{n})\right] \\
& +\nabla \cdot \mathbf{n}\left[v_{1} s_{0}-\frac{v_{1} N \kappa \xi_{s}^{2}}{D_{s}}(\delta \rho-\epsilon \nabla \cdot \mathbf{n})\right]+\frac{N \kappa C_{3}}{45 D_{r} D_{s}} \nabla \cdot \mathcal{J}=0,
\end{aligned}
$$

with the current

$$
\begin{aligned}
& \mathcal{J}=\frac{\xi_{s}^{2}\left(3 A D_{s}-v_{1} N \kappa\right)}{4 D_{s}}\left[\mathbf{n}|\nabla \rho|^{2}+\frac{1}{3} \nabla \rho(\mathbf{n} \cdot \nabla \rho)\right]-v_{1}\left(s_{0}-\frac{N \xi_{s}^{2} \kappa}{D_{s}} \delta \rho\right)(\nabla \rho \cdot \nabla) \mathbf{n} \\
& -\frac{v_{1}}{2}\left(s_{0}-\frac{N \xi_{s}^{2} \kappa}{D_{s}} \delta \rho\right) \nabla \rho \times(\nabla \times \mathbf{n})-\frac{v_{1}}{3}\left(s_{0}-\frac{N \xi_{s}^{2} \kappa}{D_{s}} \delta \rho\right)(\nabla \cdot \mathbf{n}) \nabla \rho=0
\end{aligned}
$$

The linearised equation for $\rho$ written in terms of $\mathbf{n}$ is

$$
\left[\partial_{t}-\left(D-\xi_{s}^{2} B \rho_{0}\right) \nabla^{2}\right] \rho_{0}^{-1} \rho+\left(v_{1}-\epsilon \xi_{s}^{2} B \rho_{0} \nabla^{2}\right) \nabla \cdot \mathbf{n}=0
$$

\section{APPENDIX III: LINEAR ANALYSIS AND MODE STRUCTURE IN THE SATURATED REGIME : DETAILS}

In the saturated limit the dynamical matrix takes the form

$$
\mathbf{M}_{s}=\left[\begin{array}{cc}
D q^{2}-\rho_{0} B & \rho_{0}\left(v_{0}+\epsilon \rho_{0} B\right) \\
\rho_{0} A / 3-v_{0} q^{2} / 3 \rho_{0} & \left(D+\frac{v_{0}^{2}}{30 D_{r}}\right) q^{2}+2 D_{r}^{\prime}
\end{array}\right]
$$

where $D_{r}^{\prime} \equiv D_{r}-\epsilon \rho_{0} A / 6$. The mode structure is

$$
-i \omega=\left\{\begin{array}{c}
\frac{G}{2 D_{r}^{\prime}}-\left[2 D+\frac{v_{0}\left(v_{0}+\epsilon \rho_{0} B\right)}{3 D_{r}^{\prime}}\right] q^{2}, \\
-2\left(D_{r}-\frac{\rho_{0} B}{2}\right)-\left[2 D+\frac{2 v_{0}^{2}}{45 D_{r}}-\frac{2 \epsilon N \kappa_{2} v_{0} \rho_{0}}{135 D_{r}}\left(\frac{\beta_{1}}{D_{p}}+\frac{\alpha_{1}}{D_{s}}\right)\right] q^{2},
\end{array}\right.
$$

where $G=2 \rho_{0} B D_{r}+\frac{1}{3} \rho_{0} A v_{0}$. 
Note that $G$ gives the strength and sign of effective long ranged interaction between the centres of mass of two swimmers taking into account both phoretic and chemotactic response of swimmers to $\mathrm{S}$ and $\mathrm{P}$ gradients. We see in (8.2) that the main novelty of the saturated limit is that it leads to a non-vanishing relaxation rate at $q=0$ for both modes, notwithstanding the conservation law governing $\rho$. For $D_{r}>>0$, there is a wide separation in time scale between the two modes; the first mode whose corresponding eigenvector is mostly $\rho$ relaxes slowly compared to the other which is predominantly $\mathbf{n}$. This makes it possible to make the dynamics of $\mathbf{n}$ slaved to $\rho$ and obtain a meaningful effective equation for the latter. We will show that the $\rho$ dynamics is equivalent to that of an electrolyte or a gravitational system in a passive frictional medium for $G<0$ or $G>0$ respectively.

$G<0$ implies a repulsive interaction between the swimmers; $\rho$ and $-G / 2 D_{r}^{\prime}$ in this case are analogous to the charge density and Ohmic conductivity respectively in an electrolyte. Remember that the static structure factor is simply the strength of fluctuations divided by the rate at which they decay; for $\rho$ the fluctuations are number conserving so that they go as $q^{2}$ while the relaxation is anomalously fast for wavelengths larger than a screening length due to the non local nature of the Coulomb-like interactions. To obtain the structure factors requires, of course, that we add phenomenological, gaussian, spatiotemporally white noise terms, conserving for $\rho$ and nonconserving for $\mathbf{n}_{L}$ of strength $\mathcal{P}$ and $\mathcal{N}$, to the equations of motion. The full expressions for the structure factors are

$$
\begin{aligned}
S_{\rho} & =q^{2} \frac{2 \mathcal{P}+\mathcal{N}\left(v_{0}+\epsilon \rho_{0} B\right)^{2} / 2 D_{r}^{2}}{-G / 2 D_{r}+\left[\frac{v_{0}\left(v_{0}+\epsilon \rho_{0} B\right)}{3 D_{r}}+G D / 4 D_{r}^{\prime} D_{r}\right] q^{2}+\ldots} \\
S_{n} & =\frac{2 B^{2} \mathcal{P} / D_{r}^{2}+\mathcal{N} A^{2} / 9 D_{r}^{2}}{-G / 2 D_{r}+\left[\frac{v_{0}\left(v_{0}+\epsilon \rho_{0} B\right)}{3 D_{r}}+G D / 4 D_{r}^{\prime} D_{r}\right] q^{2}+\ldots}
\end{aligned}
$$

neglecting terms that are small when $D_{r}$ is large. There is screening behaviour where fluctuations at length scales larger than

$$
\lambda_{s}=\sqrt{\frac{2 D_{r}\left[\frac{v_{0}\left(v_{0}+\epsilon \rho_{0} B\right)}{3 D_{r}}+G D / 4 D_{r}^{\prime} D_{r}\right]}{-G}}
$$

are suppressed in an essentially wavenumber independent way. Including terms of higher order in $q$ indicated in the ellipsis in (8.7) yields a density structure factor with a peak at $q \sim G^{1 / 4}$, characteristic of microphase separation. Fluctuations in $\mathbf{n}$ are suppressed and $S_{n}$ just decays.

For $G>0$, the mode structure in (8.2) shows that there is an instability for $G>0$ so that the structure factors in (8.7) have to be interpreted differently now. For wavenumbers larger than $\lambda_{s}^{-1}$ the fluctuations die off whereas for wavenumbers smaller than $\lambda_{s}^{-1}$ the system self gravitates and collapses; this is a dissipative jeans instability. $S_{n}$ also shows a peak as density gradients produced by $\rho$ tends to align $\mathbf{n}$. These effects are reminiscent of behaviour predicted for a collection of thermophoretic colloids [51].

Increasing $A$ or $B$ and thus slowing down the relaxation of $\mathbf{n}_{L}$ takes one out of the overdamped regime. The general expressions for the eigenfrequencies are

$$
-i \omega=-D_{r}^{\prime}-D q^{2} \pm \sqrt{\left(D_{r}^{\prime 2}+G\right)-\frac{v_{0}\left(v_{0}+\epsilon \rho_{0} B\right) q^{2}}{3}}
$$


which can now become oscillatory. In the underdamped limit $\mathbf{n}$ behaves like a velocity field and the systems resembles a system conserving momentum with some subtle differences discussed in context of the advective nonlinearities in the Appendix. For $G>0$ and $D_{r}^{\prime} \rightarrow 0$ we see in (8.5) that a mode structure similar to the known hydrodynamic Jeans instability is obtained with corrections due to disspation. Modes with wavenumber larger than $q^{*}$

$$
q^{*}=\sqrt{\frac{3\left(D_{r}^{\prime 2}+G\right)}{v_{0}\left(v_{0}+\epsilon \rho_{0} B\right)}}
$$

are oscillatory and the ones smaller than $q^{*}$ are too 'massive' and collapse. Note that the crossover wavenumber is given by a competition between the interaction strength $G$ and sound speed square equivalent $v_{0}\left(v_{0}+\epsilon \rho_{0} B\right)$ for our system. For $\left(G+D_{r}^{\prime 2}\right)<0$ we now have a electrolyte that shows plasma oscillations with typical frequency $\sqrt{|G|}$ as $D_{r} \rightarrow 0$. If in addition $D_{r}^{\prime}<0$ it is possible that the oscillations could become spontaneous.

The static structure factors on the stable side are

$$
\begin{aligned}
S_{\rho} & =q^{2} \frac{\mathcal{N}}{D_{r}^{\prime}+D q^{2}}+q^{2} \frac{4 D_{r}^{2} \mathcal{P}+\mathcal{N}\left(v_{0}+\epsilon \rho_{0} B\right)^{2}}{\left[\frac{2 v_{0}\left(v_{0}+\epsilon \rho_{0} B\right)}{3} q^{2}-G\right]\left(D_{r}^{\prime}+D q^{2}\right)} \\
S_{n} & =\frac{\mathcal{N}}{D_{r}^{\prime}+D q^{2}}+\frac{B^{2} \mathcal{P}+\mathcal{N} A^{2} / 9}{\left[\frac{2 v_{0}\left(v_{0}+\epsilon \rho_{0} B\right)}{3} q^{2}-G\right]}
\end{aligned}
$$

We find a structure factor for $\mathbf{n}_{L}$ with correlation length $\sim\left(D / D_{r}^{\prime}\right)^{1 / 2}$ indicating strong fluctuations towards aster formation. On approaching $D_{r}^{\prime} \rightarrow 0$, from the stable side the peak values of $S_{\rho}$ and $S_{n}$ increase as seen in (8.7). For $G>0$ and $D_{r}<0$ we have a jeans instability with a very strongly correlated hydrodynamic field; however extensive study of this interesting regime is beyond the scope of our present work. 